\title{
FOXC2 controls formation and maturation of lymphatic collecting vessels through cooperation with NFATc 1
}

\author{
Camilla Norrmén, ${ }^{1}$ Konstantin I. Ivanov, Jianpin Cheng, ${ }^{1}$ Nadine Zangger, ${ }^{4}$ Mauro Delorenzi, ${ }^{4}$ Muriel Jaquet, ${ }^{5}$ \\ Naoyuki Miura, ${ }^{6}$ Pauli Puolakkainen, ${ }^{2}$ Valerie Horsley, ${ }^{7}$ Junhao Hu, ${ }^{8}$ Hellmut G. Augustin ${ }^{8}$ Seppo Ylä-Herttuala, \\ Kari Alitalo, ${ }^{1,3}$ and Tatiana V. Petrova ${ }^{1,5}$ \\ 'Molecular Cancer Biology Program, Biomedicum Helsinki, ${ }^{2}$ Department of Surgery, Helsinki University Central Hospital, and ${ }^{3}$ Department of Pathology, Haartman Institute \\ and Helsinki University Central Hospital, University of Helsinki, 00014 Helsinki, Finland \\ ${ }^{4}$ Swiss Institute of Bioinformatics, Génopode, 1015 Lausanne, Switzerland \\ ${ }^{5}$ Division of Experimental Oncology, Multidisciplinary Oncology Center, University of Lausanne, 1066 Epalinges, Switzerland \\ ${ }^{6}$ Department of Biochemistry, Hamamatsu University School of Medicine, 43 1-3192 Hamamatsu, Japan \\ Howard Hughes Medical Institute, The Rockefeller University, New York, NY 10065 \\ ${ }^{8}$ Joint Research Division of Vascular Biology, Centre for Biomedicine and Medical Technology Mannheim, Heidelberg University and German Cancer Research Center \\ (DKFZ-ZMBH Alliance), 69120 Heidelberg, Germany \\ ${ }^{9}$ A.I. Virtanen Institute, University of Kuopio, 70211 Kuopio, Finland
}

$\mathrm{T}$ he mechanisms of blood vessel maturation into distinct parts of the blood vasculature such as arteries, veins, and capillaries have been the subject of intense investigation over recent years. In contrast, our knowledge of lymphatic vessel maturation is still fragmentary. In this study, we provide a molecular and morphological characterization of the major steps in the maturation of the primary lymphatic capillary plexus into collecting lymphatic vessels during development and show that forkhead transcription factor Foxc2 controls this process. We further identify transcription factor
NFATcl as a novel regulator of lymphatic development and describe a previously unsuspected link between NFATc1 and Foxc2 in the regulation of lymphatic maturation. We also provide a genome-wide map of FOXC2binding sites in lymphatic endothelial cells, identify a novel consensus FOXC2 sequence, and show that NFATcl physically interacts with FOXC2-binding enhancers. As damage to collecting vessels is a major cause of lymphatic dysfunction in humans, our results suggest that FOXC2 and NFATC1 are potential targets for therapeutic intervention.

\section{Introduction}

The formation of lymphatic vessels, whose main function is to drain interstitial fluid from the tissues back to the blood circulation, begins only after the blood circulation is already functional. The initial steps of lymphatic development are controlled

Correspondence to Tatiana V. Petrova: tatiana.petrova@unil.ch

K.I. Ivanov and J. Cheng's present address is Division of Experimental Oncology, Multidisciplinary Oncology Center, University of Lausanne, 1066 Epalinges, Switzerland.

V. Horsley's present address is Dept. of Molecular, Cellular, and Developmental Biology, Yale University, New Haven, CT 06520.

Abbreviations used in this paper: CEAS, cis-regulatory element annotation system; ChIP, chromatin immunoprecipitation; CsA, cyclosporine A; EMSA, electrophoretic mobility shift assay; FDR, false discovery rate; GAPDH, glyceraldehyde 3-phosphate dehydrogenase; iLEC, intestinal LEC; LEC, lymphatic endothelial cell; MAT, model-based analysis of tiling array; NFAT, nuclear factor of activated T cells; SLC, secondary lymphoid chemokine; SMA, smooth muscle actin; SMC, smooth muscle cell. by the transcription factors Sox 18 and Prox 1 and VEGF-C and its receptor VEGFR-3 (Kukk et al., 1996; Dumont et al., 1998; Wigle and Oliver, 1999; Karkkainen et al., 2004; Francois et al., 2008). Expression of Prox 1 is induced by Sox 18 in a restricted subset of endothelial cells in the embryonic jugular veins (Wigle and Oliver, 1999; Francois et al., 2008). These VEGF-Cresponsive cells sprout and migrate to form the primary lymphatic sacs, which further remodel to form a primary lymphatic capillary plexus (van der Putte, 1975; Karkkainen et al., 2004). The primary plexus undergoes further morphological and molecular changes to form a mature lymphatic network composed of

(C) 2009 Norrmén et al. This article is distributed under the terms of an AttributionNoncommercial-Share Alike-No Mirror Sites license for the first six months after the publication date (see http://www.jcb.org/misc/terms.shtml). After six months it is available under a Creative Commons License (Attribution-Noncommercial-Share Alike 3.0 Unported license, as described at http://creativecommons.org/licenses/by-nc-sa/3.0/). 
lymphatic capillaries responsible for the uptake of lymph from the interstitial space and collecting lymphatic vessels that transport the lymph back to the blood circulation. Mature collecting vessels are characterized by a thin basement membrane, sparse pericyte coverage, and have intraluminal valves to prevent backflow of the fluid. Lymphatic capillaries, however, lack valves and a pericyte layer. These later steps of lymphatic vascular development, the maturation of the primary lymphatic plexus into functional collecting vessels and capillaries, are at present not well understood.

Loss of forkhead transcription factor Foxc2 leads to defects in lymphatic remodeling, failure to form lymphatic valves, and increased pericyte coverage of lymphatic vessels (Petrova et al., 2004). In lymphedema-distichiasis (Online Mendelian Inheritance in Man reference no. 153400), a disease caused by mutations in FOXC2, the patients have a similar phenotype to Foxc $2^{-/-}$mice with abnormal lymphatic vessel pericyte coverage (Petrova et al., 2004). Lymphedema-distichiasis patients also show reflux of tracer in a lymphoscintigram, indicating failure or absence of valves in their collecting lymphatic vessels (Brice et al., 2002). Interestingly, a recent study shows linkage of FOXC2 mutations to varicose veins and primary valve failure in veins, suggesting that FOXC2 controls the formation of venous valves (Mellor et al., 2007).

The transcription factor NFATc1 belongs to a family of calcium-sensitive transcription factors activated by calcineurindependent dephosphorylation (for review see Crabtree and Olson, 2002). Activation of nuclear factor of activated $\mathrm{T}$ cells (NFAT) proteins results in exposure of a nuclear localization sequence and subsequent translocation to the nucleus. Once in the nucleus, NFATc1 interacts with other transcription factors, including AP-1, nuclear factor $\kappa \mathrm{B}$, Foxp3, and GATA, to form regulatory complexes on DNA (Kinoshita et al., 1997; Musaro et al., 1999; Macian et al., 2001; Wada et al., 2002; Sakuma et al., 2003; Bettelli et al., 2005). NFATc1 is implicated in the regulation of immune cells as well as in osteogenesis and hair follicle stem cells (Macian, 2005; Takayanagi, 2007; Horsley et al., 2008). Studies of NFATC1-deficient mice have also revealed an essential role for NFATc1 in the morphogenesis of cardiac valves (de la Pompa et al., 1998; Ranger et al., 1998).

In this study, we have characterized the steps of lymphatic vascular maturation, which lead to the formation of two morphologically and molecularly distinct parts of the lymphatic vascular system: collecting lymphatic vessels and capillaries. We show that Foxc2 controls this process, and in the absence of Foxc2, lymphatic vessels fail to undergo transformation toward a lymphatic collecting vessel phenotype. Furthermore, we demonstrate that NFATc1 is a novel regulator of lymphatic vascular maturation and that it cooperates with Foxc2 in this process.

\section{Results}

Molecular characterization of developing collecting lymphatic vessels

To characterize the process of collecting lymphatic vessel formation, we studied morphological changes and the expression of the lymphatic endothelial-specific markers VEGFR-3, Foxc2,
Prox 1, and LYVE-1 in mouse mesenteric lymphatic vessels during development. The mesenteric lymphatic primordia can first be identified at embryonic day (E) 13.5 near the splenic and superior mesenteric veins (van der Putte, 1975). At E14.5, staining for lymphatic endothelial receptor tyrosine kinase VEGFR-3 showed numerous isolated clusters or single lymphatic endothelial cells (LECs) extending long filopodia (Fig. 1 A and Fig. S1 A). These cell clusters, located on both sides along the mesenteric blood vessels, migrate and sprout toward each other along and across the blood vessels. By E15.5, LECs form a mesh-like network of highly branched capillary-like vessels surrounding the mesenteric arteries and veins (Fig. 1 B). After E15.5, the mesenteric lymphatic capillary plexus undergoes substantial morphological changes, leading to formation of collecting lymphatic vessel trunks by E17.5, characterized by even diameter, presence of intraluminal valves, and absent or little branching (Fig. 1, C and D). These features become even more pronounced in the postnatal collecting vessels (Fig. $1 \mathrm{E}$ ).

On the molecular level, E14.5-15.5 lymphatic vessels express uniformly high levels of Prox1 and VEGFR-3 (Fig. 1, A and K). At E14.5, LYVE-1 expression was absent in lymphatic vessels in the skin, whereas the lymph sacs show high LYVE-1 expression (Fig. S1, B-E). Interestingly, in the mesenterium, only a small subset of LECs express LYVE-1 at this time point (Fig. S1, G and L). At E14.5, Foxc2 is mainly expressed in arterial smooth muscle cells (SMCs), and Foxc2 levels are high only in a few isolated LECs (Fig. 1 F). Strikingly, by E15.5, all LECs express high levels of Foxc2 (Fig. 1 G). Up-regulation of Foxc 2 expression is followed by an increase in LYVE-1 levels, which becomes present in all LECs by E16.5 (Fig. S1, H-N). Between E15.5 and 16.5, the expression of first Foxc2 and then Prox 1 begins to decrease except in the areas of characteristic constrictions, which delineate forming lymphatic valves, and by E17.5, only weak expression can be detected in the lymphangion segments between the valves (Fig. 1, G-J, L-O, and Q-T). Foxc2 and Prox1 down-regulation is followed by a decrease of VEGFR-3 and LYVE-1 at E17.5-postnatal day (P) 9, but VEGFR-3 remains high in the valve leaflets, whereas LYVE-1 expression becomes very low in all collecting LECs, as it has been reported previously (Fig. 1, D and E; and Fig. S1, J, K, O, and P; Makinen et al., 2005). In the fully mature postnatal collecting lymphatic vessels, the expression of Foxc2, Prox1, and VEGFR-3 in vessel trunks is even weaker, whereas the expression in the valves remains high.

The presence of a thin basement membrane and a sparse coating with mural cells is another characteristic feature of collecting lymphatic vessels. We found that accumulation of basement membrane protein collagen IV starts at E16.5 (Fig. 2, A-J). NG2-positive mural cells start to appear around the forming collecting vessels at E17.5, whereas $\alpha$ smooth muscle actin (SMA)-positive SMCs are prominent only after birth (Fig. 2, A-E and $\mathrm{K}-\mathrm{X})$.

In summary, the formation of lymphatic collecting vessels starts at E14.5-15.5, which is coincident with up-regulation of Foxc2 (Fig. $1 \mathrm{U}$ ). This is followed by remodeling of the primary capillary plexus and formation of a single lymphatic trunk, at which point the expression of Foxc 2 decreases except in the areas 

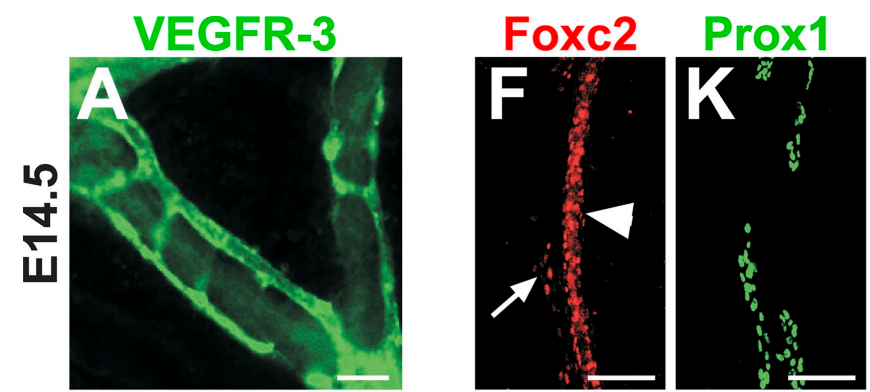

merge
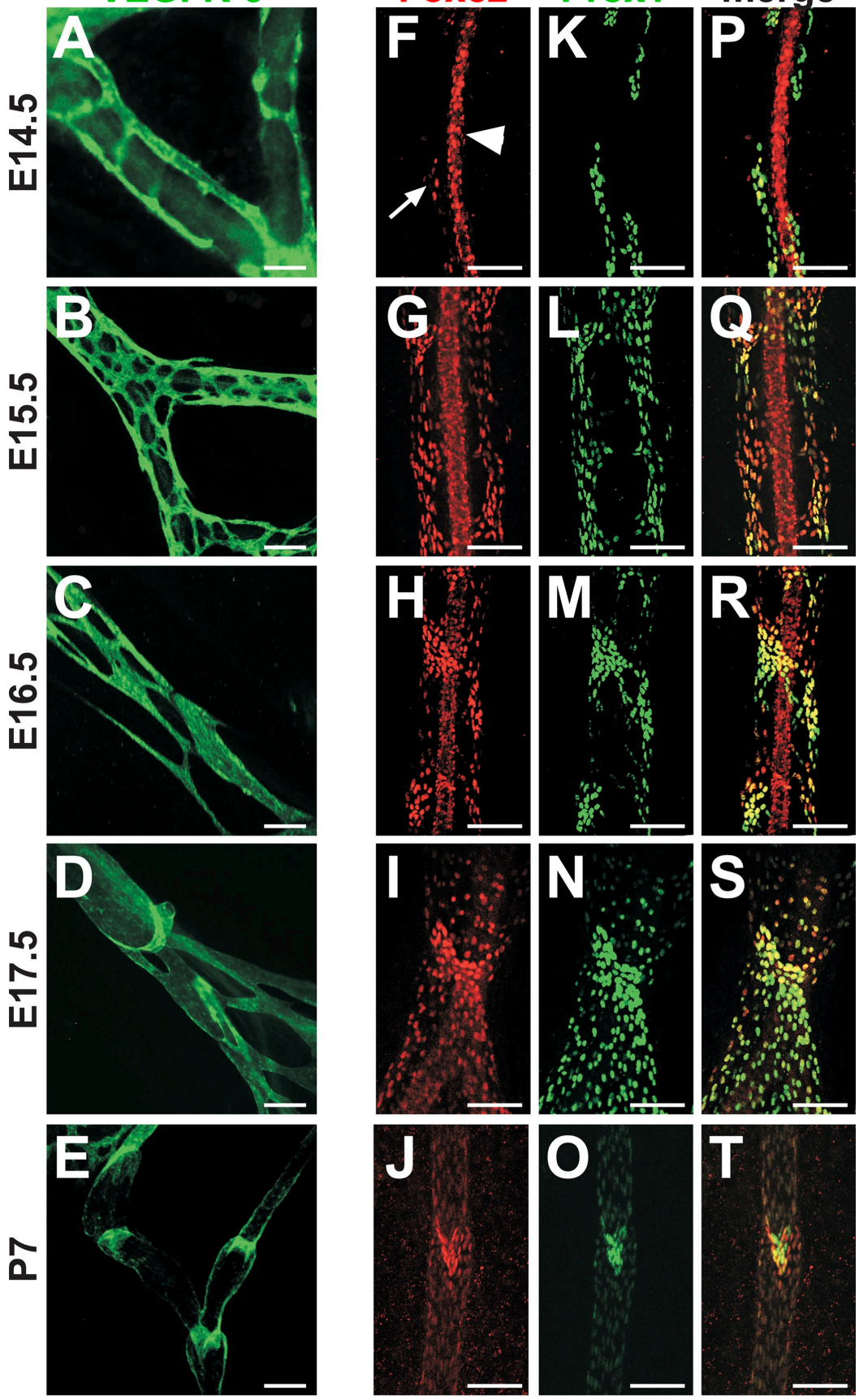

$\mathrm{U}$

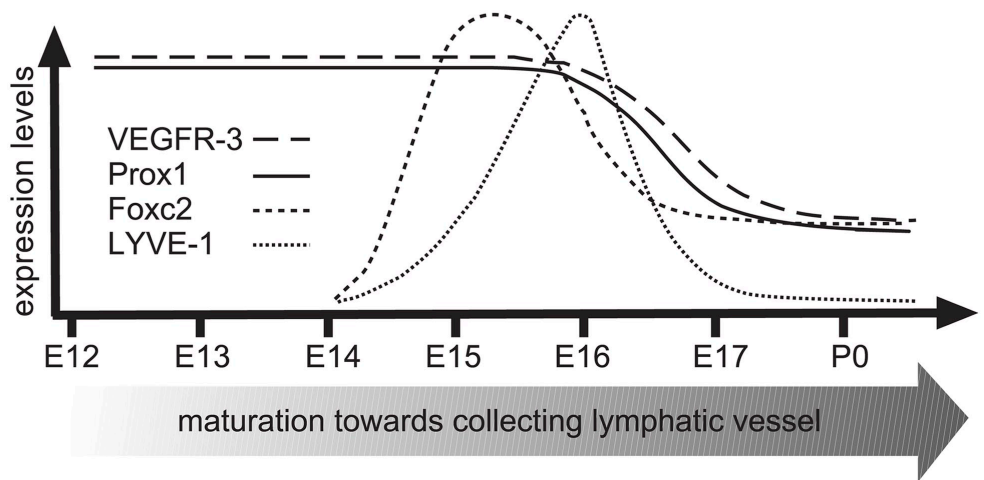



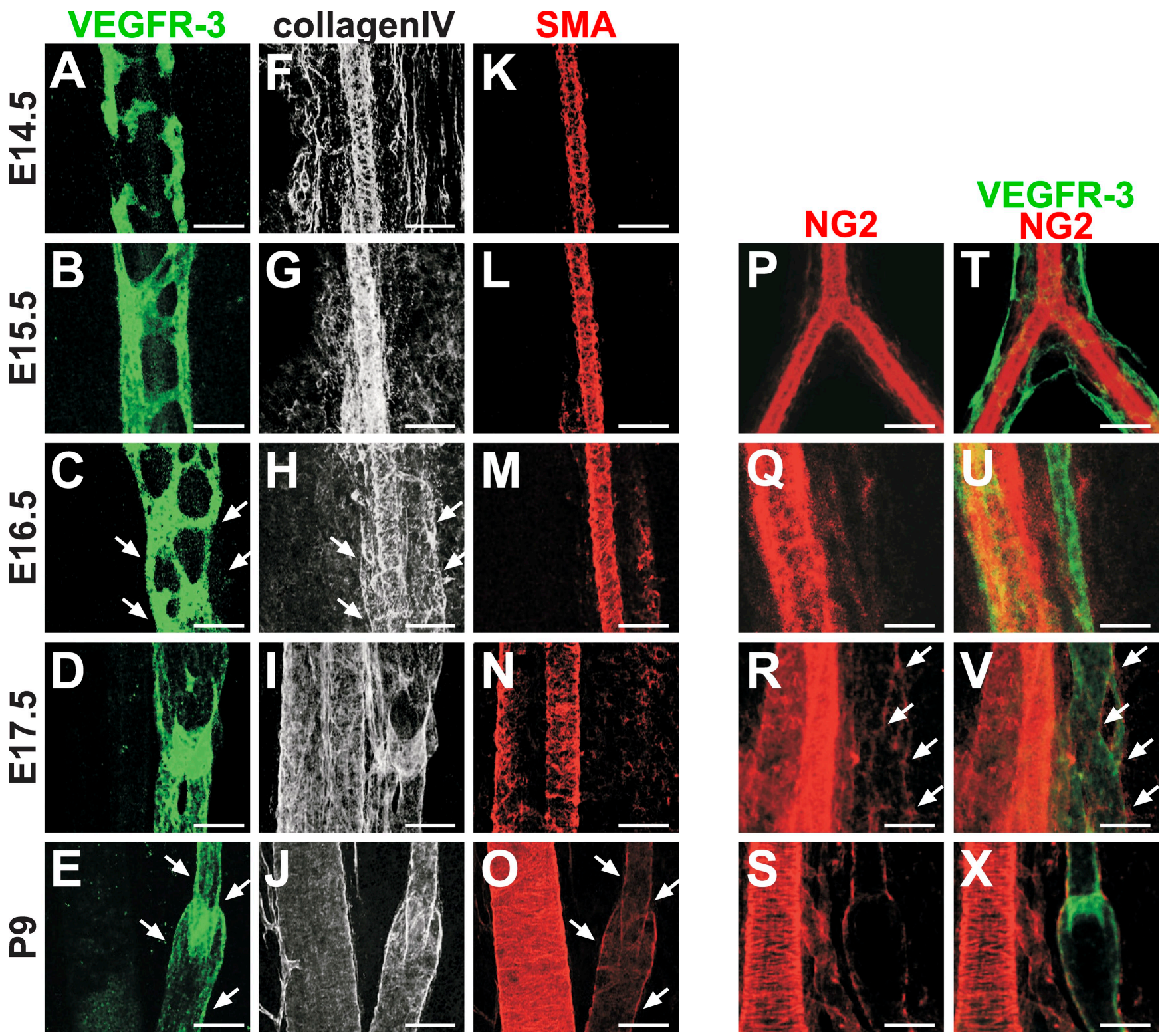

Figure 2. Formation of basement membrane and mural cell coating during maturation of lymphatic collecting vessels. (A-X) Whole-mount immunofluorescent staining of wild-type mesenteric vessels at the indicated ages for VEGFR-3 (A-E), collagen IV (F-J), SMA (K-O), and NG2 (P-X). Accumulation of basement membrane to collecting lymphatic vessels occurs at E16.5 (C and H, arrows), whereas recruitment of SMCs occurs between E17.5 and P9 ( $E$ and $O$, arrows), and NG2-positive mural cells appear around the vessels at E17.5 (R and V, arrows). Bars, $100 \mu \mathrm{m}$.

of the forming lymphatic valves, and is further followed by a decrease in Prox1, VEGFR-3, and LYVE-1 expression. Accumulation of basement membrane proteins starts at E16.5 followed by recruitment of NG2-positive mural cells at E17.5 and SMCs postnatally. These data uncover a highly dynamic spatiotemporal expression profile for LEC markers during the formation and maturation of collecting lymphatic vessels and suggest that the change in Foxc2 expression is the critical first event of the maturation process.

Loss of Foxce prevents formation of collecting Iymphatic vessels

Up-regulation of Foxc2 at E15.5, which precedes the morphological and molecular changes in the maturing lymphatic network, suggested that Foxc2 controls lymphatic vascular maturation. Indeed, in Foxc $2^{-/}$mice, lymphatic vessels fail to downregulate Prox1, VEGFR-3, and LYVE-1 (Fig. 3). In contrast, the collecting vessels in the mesentery of E17.5 heterozygous littermates show a normal expression pattern with low levels of VEGFR-3 and Prox1 in the lymphangion, high expression in the valves (Fig. 3, A and E), and uniformly low expression of LYVE-1 throughout the whole collecting vessel (Fig. 3 G). The lymphatic capillaries continue to express high levels of LYVE-1 both in Foxc2 $2^{-/-}$mice and in heterozygous littermates (Fig. 3, $\mathrm{G}$ and $\mathrm{H}$ ). The secondary lymphoid chemokine (SLC) is also highly expressed in the immature collecting vessels in Foxc2 $2^{-/-}$ mice, whereas it is present only in the valves of the collecting vessels of $\mathrm{Foxc}^{+/+}$mice (Fig. 3, I and J). In addition, the 

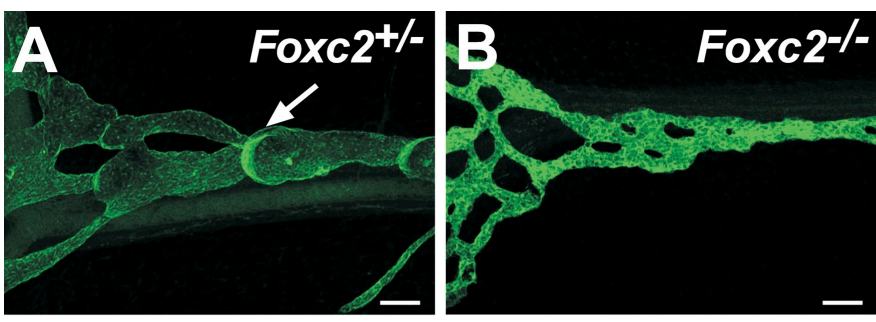

VEGFR-3
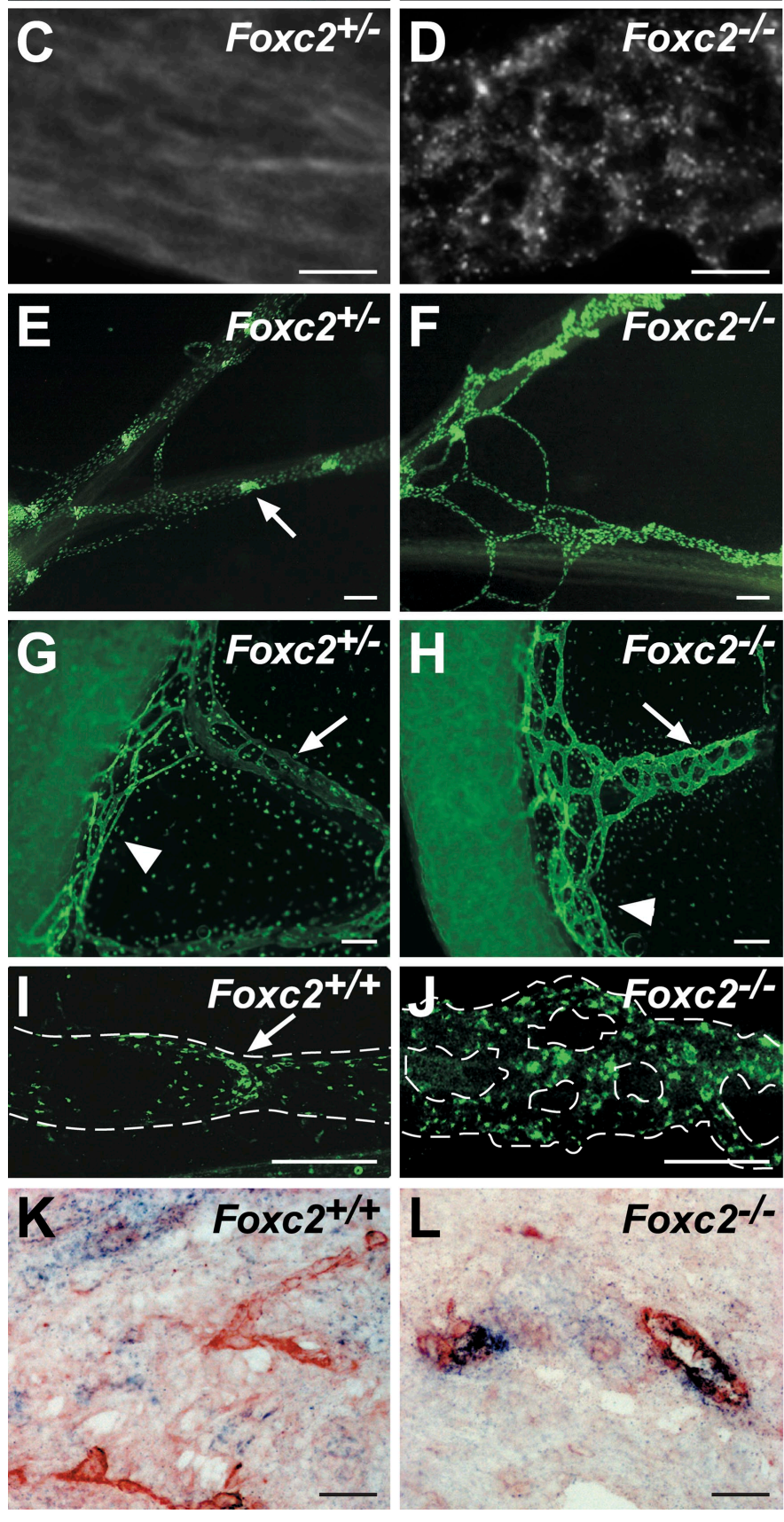

\section{Prox1}

LYVE-1

\section{Ang2 ISH}

Figure 3. Foxc2 is essential for the formation of collecting lymphatic vessels. (A-J) Mesenteric lymphatic vessels fail to down-regulate VEGFR-3, Prox 1, and SLC in the absence of Foxc2. Whole-mount immunofluorescent staining for VEGFR-3 (A-D), Proxl (E and F), LYVE-1 (G and H), and SLC (l and J) in E17.5 Foxc2 ${ }^{-1-}$ or control embryos. (C and D) Internalization of VEGFR-3 in lymphatic vessels of Foxc2 $2^{-/-}$ mice but not control E17.5 embryos. (K and L) Increased Angpt2 expression in lymphatic vessels of Foxc2 $2^{-1-}$ mice. In situ hybridization for Angpt2 (blue) and immunohistochemical staining for VEGFR-3 (red). Arrows, lymphatic valves (A, E, and I) or collecting lymphatic vessels ( $G$ and $H)$; arrowheads, lymphatic capillaries; dashed lines, lymphatic vessels. Bars: $(A, B$, and $\mathrm{E}-\mathrm{J}) 100 \mu \mathrm{m} ;(\mathrm{C}, \mathrm{D}, \mathrm{K}$, and L) $25 \mu \mathrm{m}$.

VEGFR-3 IHC

collecting vessels in Foxc $2^{-/-}$mice are more highly branched, which may reflect incomplete intussusceptive vascular pruning (Burri et al., 2004).

Activation of VEGFR-3 after binding to its ligand VEGF-C is followed by internalization of the receptor observed in cultured LECs and in sprouting lymphatic capillaries in vivo (Makinen et al., 2001; Veikkola et al., 2001; Wirzenius et al., 2007).
We observed uniform membranous staining for VEGFR-3 in wildtype collecting vessels, which is consistent with a quiescent VEGF-C-VEGFR-3 pathway (Fig. 3 C). In contrast, VEGFR-3 in Foxc $2^{-/-}$mice was concentrated in grainy dots and largely absent from the cell surface, which is similar to sprouting lymphatic capillaries (Fig. 3 D and Fig. S1 F). Although the exact reason for the increased VEGFR-3 internalization remains to be investigated, it 
is indicative of active VEGFR-3 signaling and further confirms the failure of Foxc $2^{-/}$lymphatic vessels to adopt a collecting vessel phenotype. Angiopoietin-2 (Ang2 and Angpt2), a ligand of Tie 2 endothelial receptor tyrosine kinase, is important in the regulation of blood vascular maturation and stabilization but is also essential for normal lymphatic development. Angpt2-deficient mice display disorganized and hypoplastic lymphatic capillaries and an aberrant recruitment of periendothelial cells to lymphatic capillaries (Gale et al., 2002; Shimoda et al., 2007; Dellinger et al., 2008). Ang2 is produced by LECs in vivo (Fig. S2 F; Dellinger et al., 2008), and its expression is induced in cultured LECs upon treatment with VEGF-C (Veikkola et al., 2003). In agreement with the increased VEGFR-3 internalization, we observed higher levels of Angpt 2 in Foxc2-deficient lymphatic endothelia (Fig. 3, K and L). Activation of VEGFR-3 also leads to enhanced sprouting of lymphatic capillaries, accompanied by formation of filopodia (Saaristo et al., 2002a; Wirzenius et al., 2007). We observed abundant lymphatic sprouting in the developing lymphatic vasculature in the skin of wild-type mice (Fig. 4, A-E). In contrast, Foxc $2^{-/-}$lymphatic vessels contained numerous blunt-ended protrusions devoid of filopodia, which were closely associated with a surrounding layer of basement membrane protein collagen IV (Fig. 4, F-J).

Collectively, our data show that loss of Foxc2 leads to a failure in the maturation of the primary lymphatic plexus into functional collecting lymphatic vessels and capillaries. Instead, the Foxc $2^{-/-}$lymphatic vasculature is characterized by a persistent expression of the lymphatic capillary markers Prox1, VEGFR-3, LYVE-1, and SLC, failure to down-regulate VEGFR-3 signaling, and abnormal capillary sprouting.

\section{NFATC1 is expressed in LECs and is controlled by PROX1 and VEGF-C}

Failure to form intraluminal valves is a characteristic feature of Foxc $2^{-/-}$lymphatic vessels (Petrova et al., 2004), which led us to investigate the potential for cooperation between Foxc 2 and pathways implicated in the regulation of heart valve development such as Wnt and NFAT (Armstrong and Bischoff, 2004). We did not observe $\beta$-galactosidase expression in the lymphatic valves of the Wnt reporter mice TOP-Gal or BAT-Gal, whereas strong LacZ staining was observed in cardiac valves as reported previously (DasGupta and Fuchs, 1999; Maretto et al., 2003; unpublished data). Similarly, reporter expression was not modified in the absence of Foxc2 in TOP-Gal;Foxc $2^{-1-}$ or BAT$\mathrm{Gal} ;$ Foxc $2^{-/-}$mice (unpublished data).

However, for NFATc1, we detected previously unsuspected expression and activity in the lymphatic vasculature. At E12.5, nuclear NFATc1 was present in LECs of the lymph sacs. At E13.5, NFATc1 was mainly detected in the cytoplasm and, at lower levels, in the nuclei of endothelial cells of the lymph sacs. Mostly nuclear localization was observed from E14.5 onwards (Fig. 5, A-H). Notably, no significant expression of NFATc1 was detected in blood vessels at E12.5-17.5 nor in other Prox1expressing tissues, such as the spinal cord (Fig. S2, B and C; unpublished data), suggesting a LEC-specific function in addition to the previously described role in endocardial cells (de la Pompa et al., 1998; Ranger et al., 1998; Chang et al., 2004).
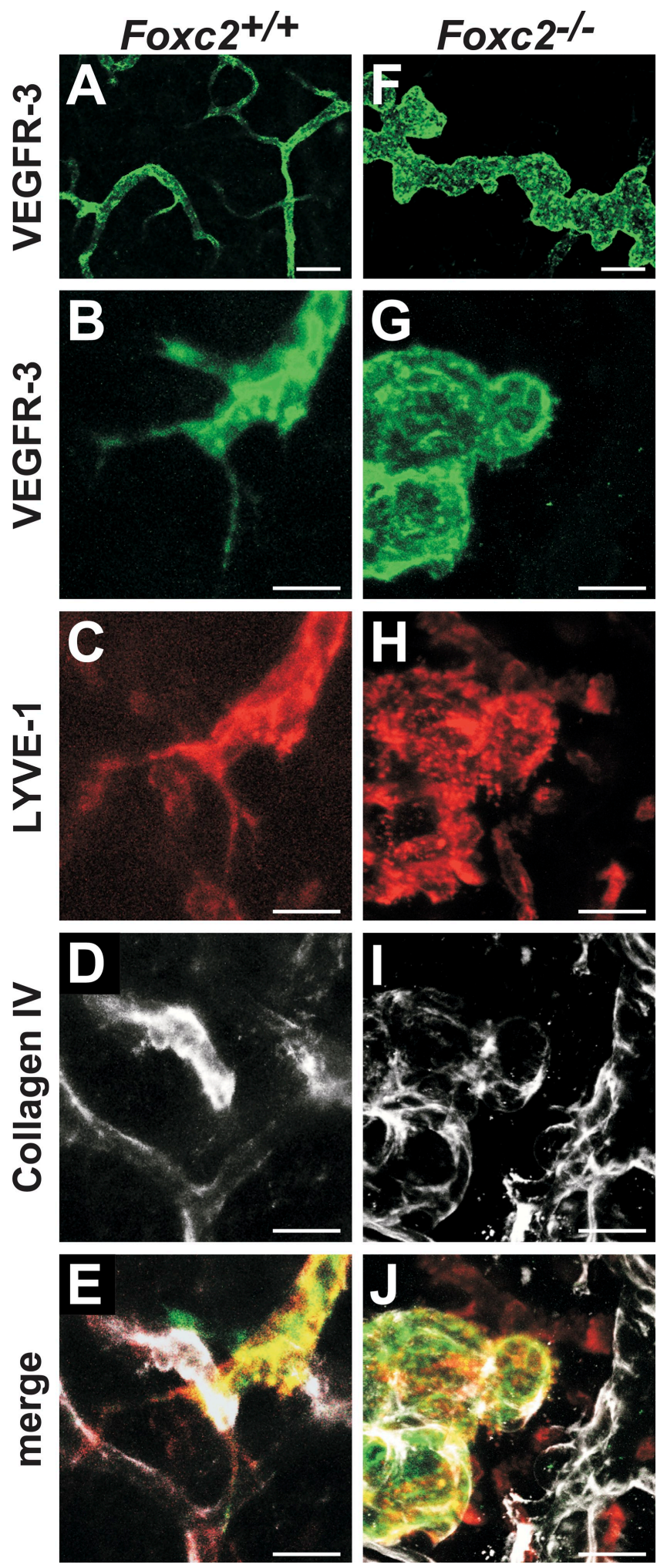

Figure 4. Defective sprouting of lymphatic vessels in Foxc2-/- mice. (A-J) Whole-mount immunofluorescent staining for VEGFR-3 (green), LYVE-1 (red), and collagen IV (white) of skin from E17.5 wild-type and Foxc2 $2^{-1}$ embryos. Note the close association of collagen IV and aborted sprouts in Foxc2 $2^{-1-}$ lymphatic vessels (I and J), whereas no collagen is deposited around wild-type lymphatic vessels (D and E). Bars: (A and F) $100 \mu \mathrm{m}$; (B-E and G-J) $50 \mu \mathrm{m}$. 

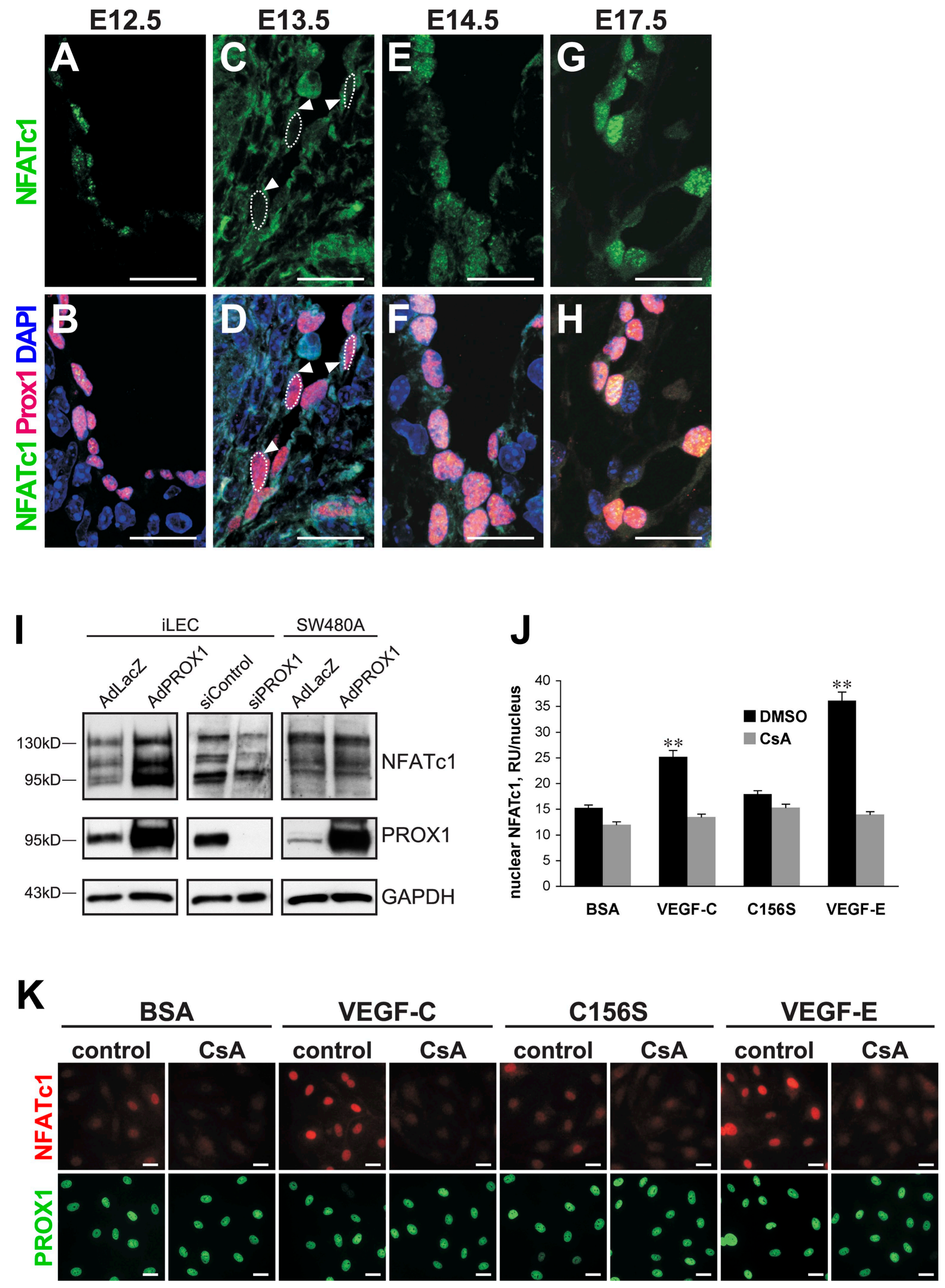

Figure 5. Expression and regulation of NFATc1 in LECs. (A-H) Immunofluorescent staining for NFATcl (green), Prox 1 (red), and DNA (blue) at E12.5-17.5. Arrowheads, cytoplasmic staining. (I) Western blotting for the indicated proteins of iLECs transfected with PROXI siRNA, control siRNA, or iLECs and control cell line SW480A (a subclone of colon carcinoma cell line) transduced with AdPROXI or control AdLacZ. (J and K) NFATcl activation is VEGF-C and VEGFR-2 dependent. Cells were stimulated with the indicated growth factors or BSA in the presence and absence of CsA and stained for NFATc 1 (red) and PROXI (green). (J) Quantification of nuclear intensity of NFATcl in iLECs. Data are presented as mean percentual nuclear intensity \pm SEM. $* *, P<0.001 . R U$, relative intensity unit. Bars, $20 \mu \mathrm{m}$. 
The in situ hybridization analysis demonstrated expression of NFATCl in lymphatic vessels, especially in the valves, further confirming our observations (Fig. S2, D-F). A more detailed analysis of Foxc 2 and NFATc1 expression revealed that these transcription factors are present only in a subset of LECs and are largely coexpressed as determined by double staining (Fig. S2 G). Moreover, in embryonic and adult skin, Foxc2 expression is mainly detected in lymphatic collecting vessels, but not in capillaries, further confirming that Foxc2 is a regulator of collecting vessel identity (Fig. S2, H-J; unpublished data).

Because of the specific expression of NFATc1 in lymphatic but not blood vascular endothelial cells, we investigated the potential regulation of NFATc1 expression by PROX1, which controls LEC commitment (Petrova et al., 2002; Wigle et al., 2002). Indeed, overexpression of PROX1, using adenoviral gene transfer in primary human intestinal LECs (iLECs), increased NFATc1 levels, whereas PROX1 overexpression in nonendothelial cells did not have any effect on NFATc1 expression (Fig. 5 I). Consistently, siRNA-mediated knockdown of PROX1 resulted in decreased NFATc1 levels (Fig. 5 I).

VEGF stimulates nuclear translocation of NFATc1 and increases proliferation in human pulmonary valve endothelial cells (Johnson et al., 2003). This led us to investigate whether VEGF-C, which has been shown to promote the formation of collecting vessels in addition to its role in lymphatic endothelial proliferation and sprouting (Tammela et al., 2007), can activate NFATc1 in LECs. Indeed, treatment of iLECs with VEGF-C resulted in strong accumulation of nuclear NFATc1, whereas control BSAtreated cells showed only little nuclear NFATc1 staining (Fig. 5, $\mathrm{J}$ and K). VEGF-E, a viral homologue of VEGF that signals through VEGFR-2 (Ogawa et al., 1998; Meyer et al., 1999), also increased nuclear NFATc1, whereas VEGF-C156S, a mutant form of VEGF-C that unlike wild-type VEGF-C does not bind to VEGFR-2 but only to VEGFR-3 (Joukov et al., 1998), did not have a marked effect on nuclear accumulation of NFATc1 in spite of the activation of ERK1/2 phosphorylation (Fig. 5, J and K; and Fig. S3 A). In contrast to its effect on nuclear translocation of NFATc1, VEGF-C treatment did not increase NFATC1 mRNA levels (Fig. S3 B), and conversely, overexpression of PROX1 in LECs did not lead to a specifically nuclear increase in NFATc1 but rather to increased overall protein levels (Fig. S3 C). Collectively, our data indicate that NFATc1 is expressed in a subset of LECs and that its levels are controlled by PROX1, whereas its activity and nuclear translocation are regulated by VEGF-C through activation of VEGFR-2 or VEGFR-2-VEGFR-3 heterodimers.

\section{Inhibition of NFAT signaling prevents \\ formation of collecting lymphatic vessels}

Next, we studied whether inhibition of NFATc1 signaling affects lymphatic vascular development in vivo. The initial LEC commitment was not affected in mice deficient in Nfatcl, as determined by staining for Prox1 (unpublished data). However, lymphatic capillaries in mice surviving until E15.5 were abnormally patterned, tortuous, and widened, which is reminiscent of the lymphatic vessels in Foxc $2^{-l-}$ mice (Fig. 6, A and B). These data suggested that NFATc1 controls the remodeling steps of lymphatic vascular development. Because $\mathrm{Natcl}^{-1-}$ mice die before the formation of fully mature collecting lymphatic vessels (de la Pompa et al., 1998; Ranger et al., 1998), we treated mice with cyclosporine A (CsA), an inhibitor of calcineurin activity, and thus dephosphorylation and nuclear translocation of NFAT transcription factors (Graef et al., 2001). CsA treatment at E11 reproduces the cardiac valve defects seen in $\mathrm{Nfatcl}^{-/-}$ mice, whereas CsA administration at E12 does not affect the heart valves or the viability of the embryos (Chang et al., 2004). To avoid interference with heart development, we treated wildtype and Foxc $2^{+--}$pregnant females at E12.5-16.5 and analyzed the vasculature at E17.5. As expected (Chang et al., 2004), CsA reduced the nuclear localization of NFATc1 in LECs (Fig. S2, $\mathrm{K}-\mathrm{N}$ ), but it did not affect the viability and overall appearance of the embryos (Fig. 6 C). Similarly, the blood vasculature of CsA-treated embryos did not differ from that of the control mice and displayed normal formation of veins, arteries, and capillary bed, appropriate coverage with SMCs, and normal blood vascular density (Fig. 6, D-K; unpublished data).

In contrast, CsA treatment resulted in abnormal lymphatic vessel patterning, lymphatic vessel hyperplasia, defective remodeling of mesenteric collecting vessels, and recruitment of SMCs to dermal lymphatic capillaries (Fig. 7, A-P and Q; and Fig. 8, A-H), which are phenotypes consistent with the early remodeling defects observed in $\mathrm{Nfatcl}^{-1-}$ mice and reminiscent of the phenotype of Foxc $2^{-/-}$mice. These defects were especially pronounced in the lymphatic vessels of CsA-treated Foxc $2^{+/-}$embryos (Fig. 7, K-P). Furthermore, the lymphatic vessels of CsA-treated mice failed to down-regulate VEGFR-3, Prox 1 (Fig. 7, A-P), and LYVE-1 (unpublished data), and CsAtreated $\mathrm{Foxc}^{+/-}$mice completely lacked lymphatic valves, whereas CsA-treated wild-type mice had a significantly reduced number of valves (Fig. 7, F, N, P, and R). Similar to lymphatic vessels in Foxc $2^{-/-}$mice, we also observed increased internalization of VEGFR-3 (Fig. 8, I-L) and higher Angpt2 levels (Fig. 8, $\mathrm{M}$ and $\mathrm{N}$ ). The blunt aborted sprouts seen in the lymphatic capillaries of $\mathrm{Foxc2}^{-/-}$mice were also present in CsA-treated Foxc $2^{+/-}$mice (Fig. 7 O). Injection of FITC-dextran into the hind limbs of E17.5 Foxc2 $2^{+-}$CsA-treated embryos demonstrated abnormal outflow from the collecting lymphatic vessels to the precollector vessel branches, whereas only the collecting lymphatic vessel trunks were visualized in control embryos, further confirming loss of collecting vessel function (Fig. 7, $\mathrm{S}$ and $\mathrm{T})$. Foxc2 was present in all LECs of CsA-treated mice, demonstrating that NFAT signaling does not control Foxc2 expression or protein stability (Fig. S2, O and P), thus, the lymphatic defects are not caused by the lack of Foxc2. Conversely, Foxc $2^{-/-}$mice showed normal NFATc1 expression (unpublished data).

Collectively, these data show that suppression of NFATc1 activation during the late stages of embryogenesis prevents the formation and maturation of collecting lymphatic vessels without affecting blood vascular development in a manner highly reminiscent of the Foxc 2 knockout phenotype. Moreover, loss of one Foxc2 allele strongly potentiates the effects of NFAT pathway inhibition. Together with the colocalization of Foxc2 and NFATc1 in developing collecting vessels, this suggests a functional cooperation between these two signaling cascades. 

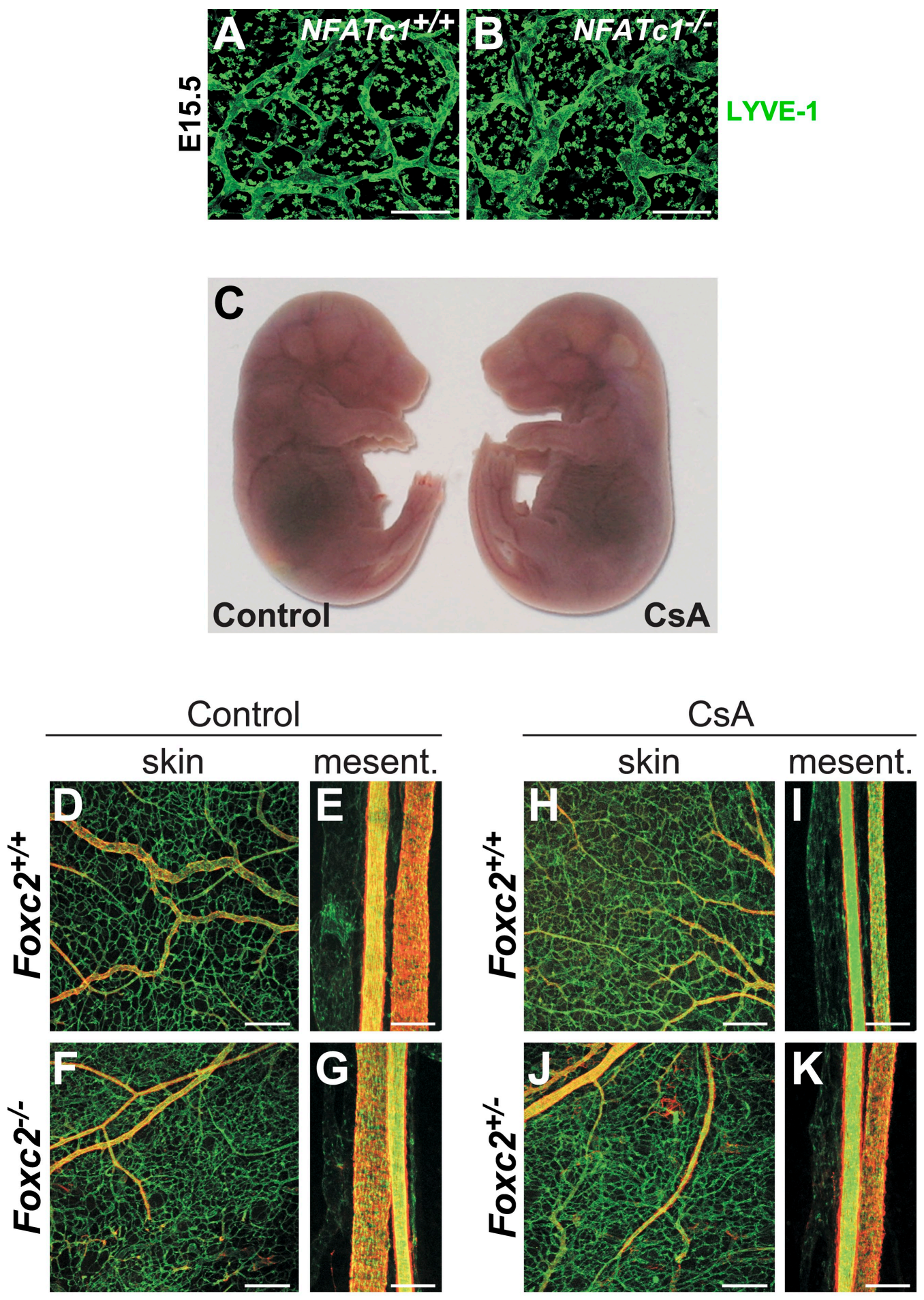

PECAM-1 SMA

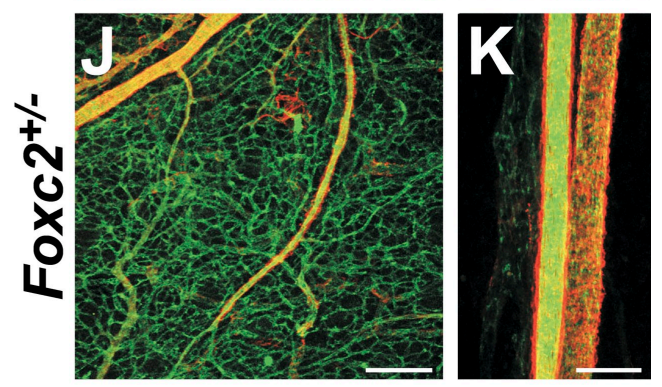

Figure 6. Defective lymphatic and normal blood vessel development upon loss of NFAT signaling. (A and B) Abnormal lymphatic vessel patterning in NFATC 1 ${ }^{-1-}$ embryos. Whole-mount immunofluorescent staining of E15.5 skin for LYVE-1. Bars, $200 \mu \mathrm{m}$. (C) Normal macroscopic appearance of E17.5 embryos after administration of CsA. (D-K) Normal blood vessel morphology and SMC coverage after administration of CsA. Whole-mount immunofluorescent staining for PECAM-1 (green) and SMA (red) of control and CsA-treated skin and mesenteric vessels of E17.5 wild-type, Foxc2 $2^{+/-}$, and Foxc2 ${ }^{-/-}$

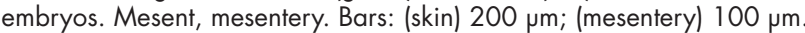

ChIP-chip analysis of FOXC2-binding sites reveals cooperation with NFAT signaling

The similarities between the lymphatic maturation defects in Foxc2-deficient mice and mice with inhibited NFATc1 signal- ing led us to investigate a possible link between FOXC2 and NFATc1. FOXC2 and NFATc1 coimmunoprecipitated, indicating that the two transcription factors physically interact at least when overexpressed (Fig. S3 D). To gain a mechanistic 


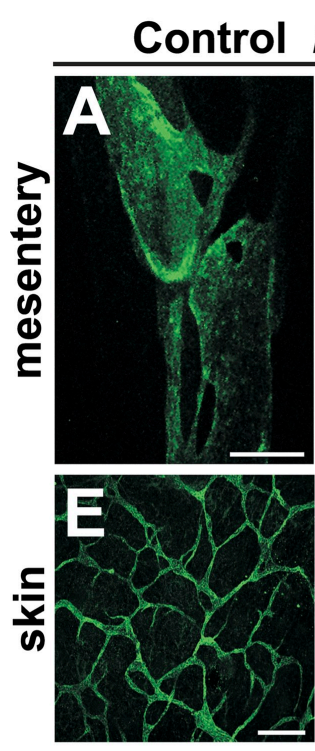

VEGFR-3

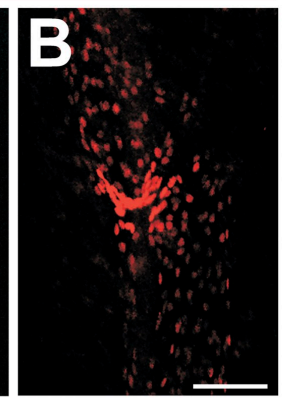

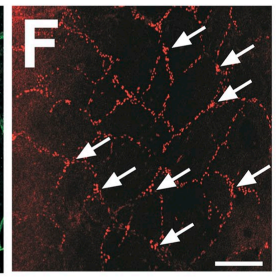

Prox1

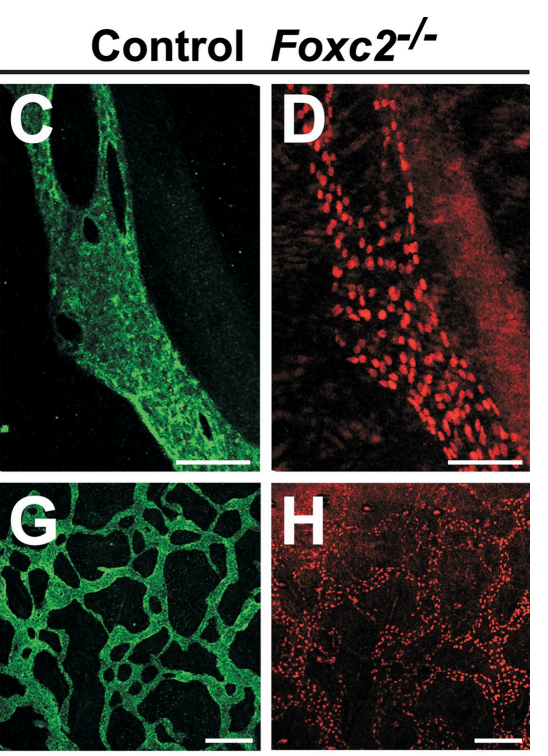

VEGFR-3

Prox1

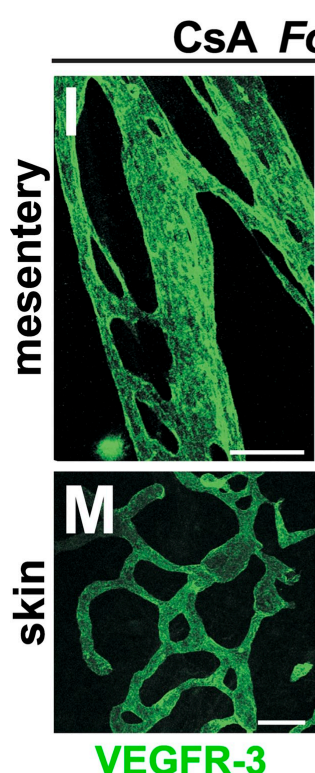

oxc2 $2^{+/+}$
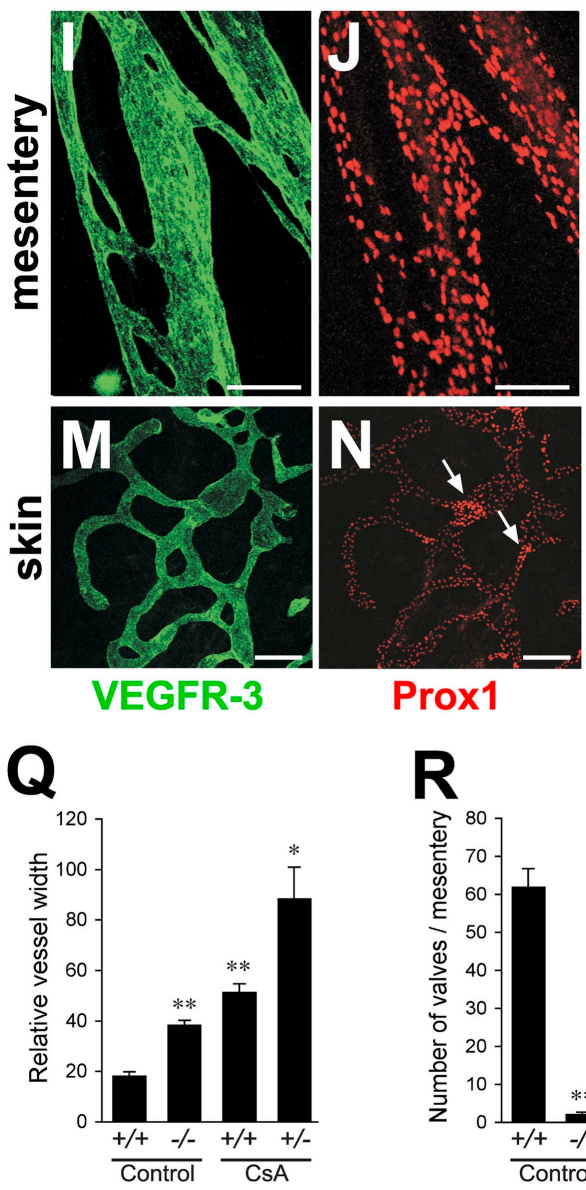
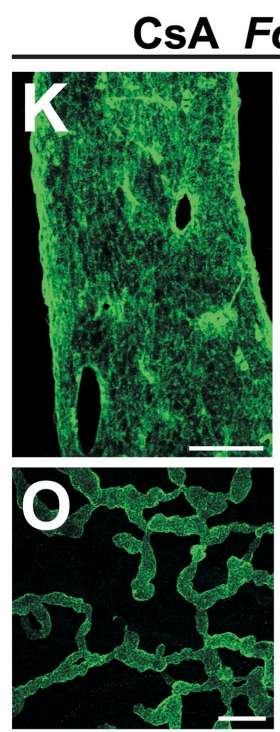

VEGFR-3
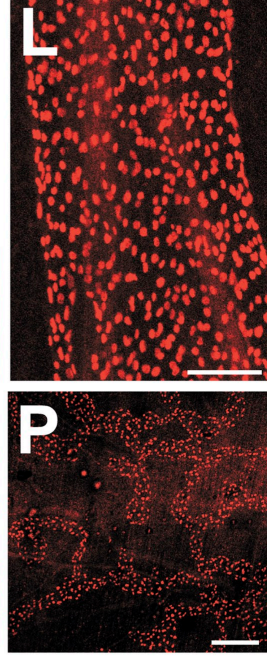

Prox1
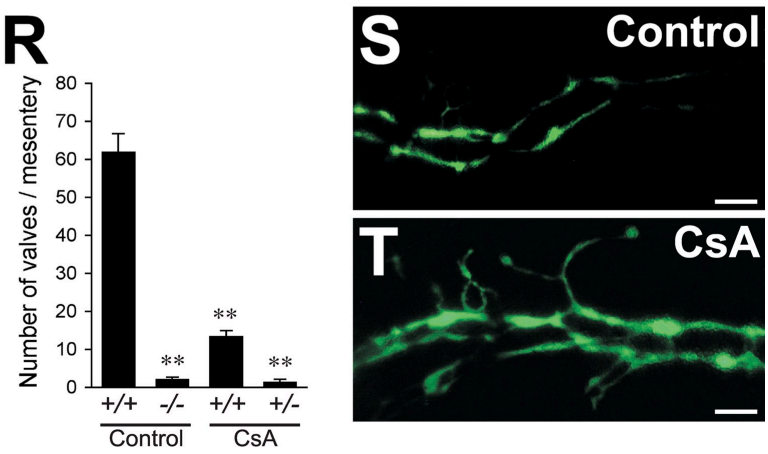

Figure 7. NFAT signaling is necessary for the formation of collecting lymphatic vessels. (A-P) NFAT inhibition prevents maturation of lymphatic vessels. Whole-mount immunofluorescent staining for VEGFR-3 (green) and Proxl (red) of mesenteries and skins of control (A-H) or CsA-treated (I-P) E17.5 embryos. Arrows, lymphatic valves. (Q) Quantification of mean lymphatic vessel width from control and CsA-treated E17.5 dermal lymphatic capillaries $(n=4)$. (R) Quantification of mesenteric lymphatic valves. $n=4$ per genotype. ${ }^{*}, \mathrm{P}<0.005 ;{ }^{*}{ }^{*}, \mathrm{P}<0.001$. (S and T) Lymph backflow in CsA-treated embryos. Collecting lymphatic vessels were visualized after high molecular weight FITC-dextran injection into E17.5 embryos. Data are presented as mean \pm SEM. Bars: (A-D and J-L) $100 \mu \mathrm{m}$; (E-H and M-P) $200 \mu \mathrm{m}$; (S and T) $500 \mu \mathrm{m}$. 


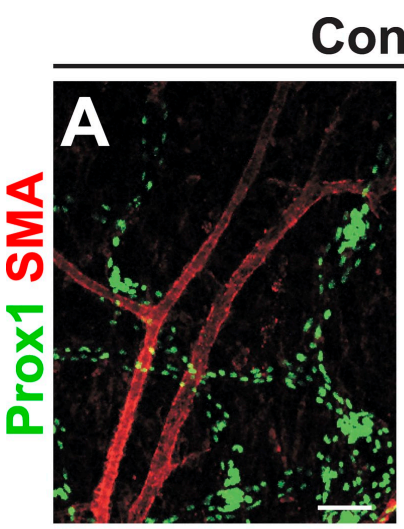

Control
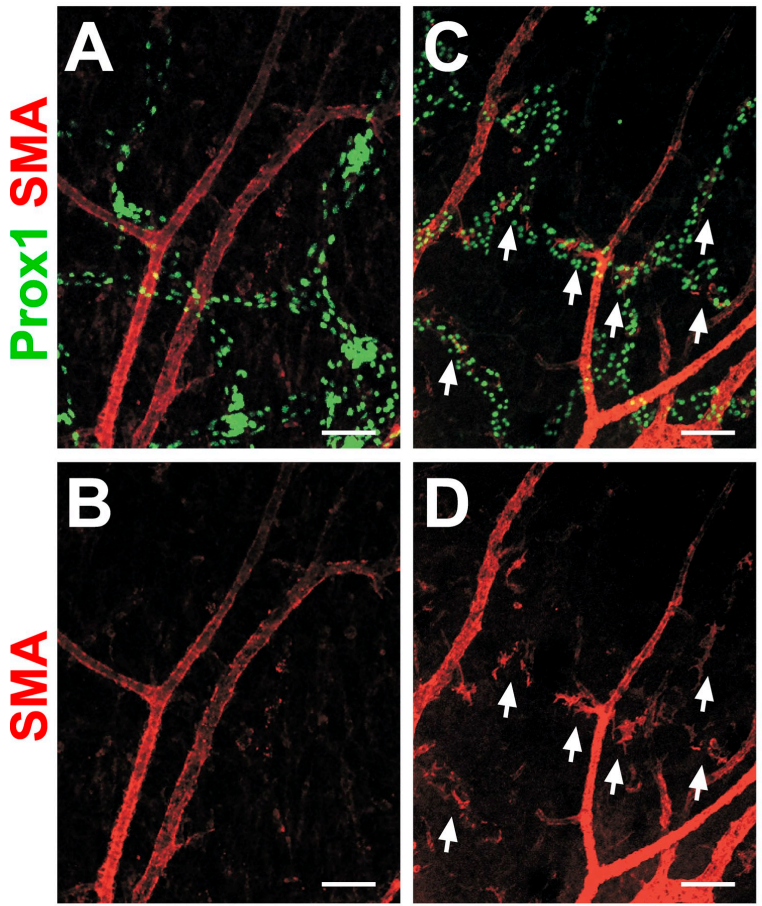

$\mathrm{FoxC2}^{+/-}$

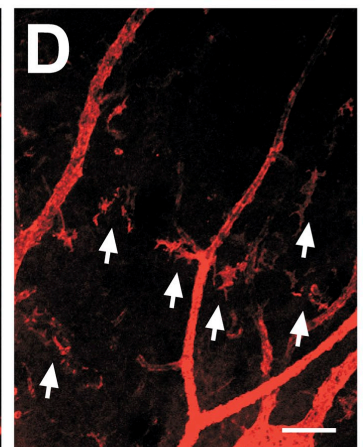

Foxc2 $2^{-/-}$

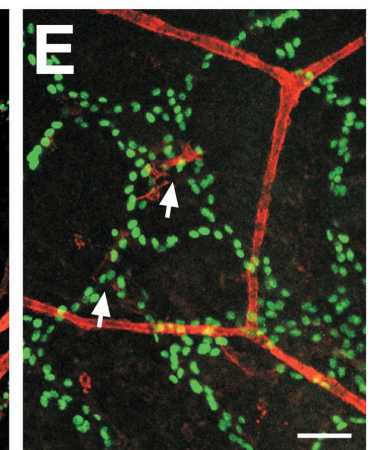

CsA

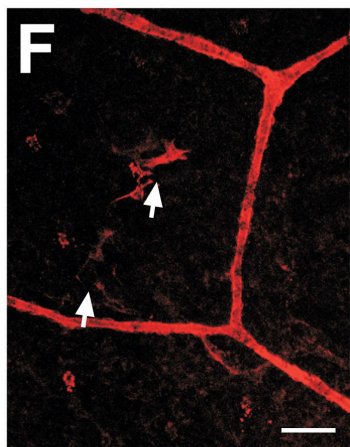

Foxc2 $^{+/+}$
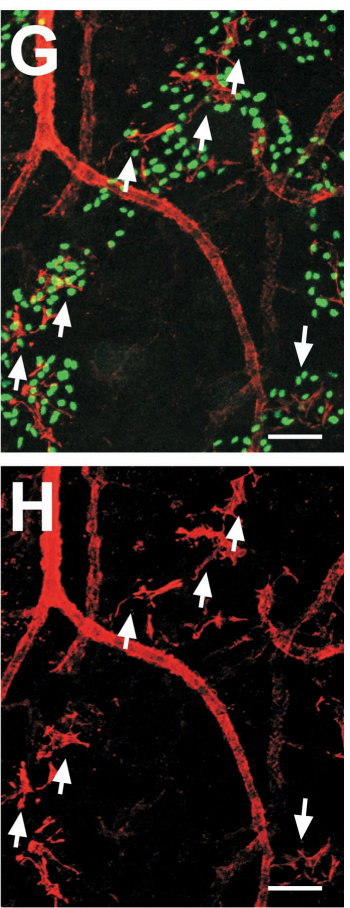

Foxc2 $^{+/-}$
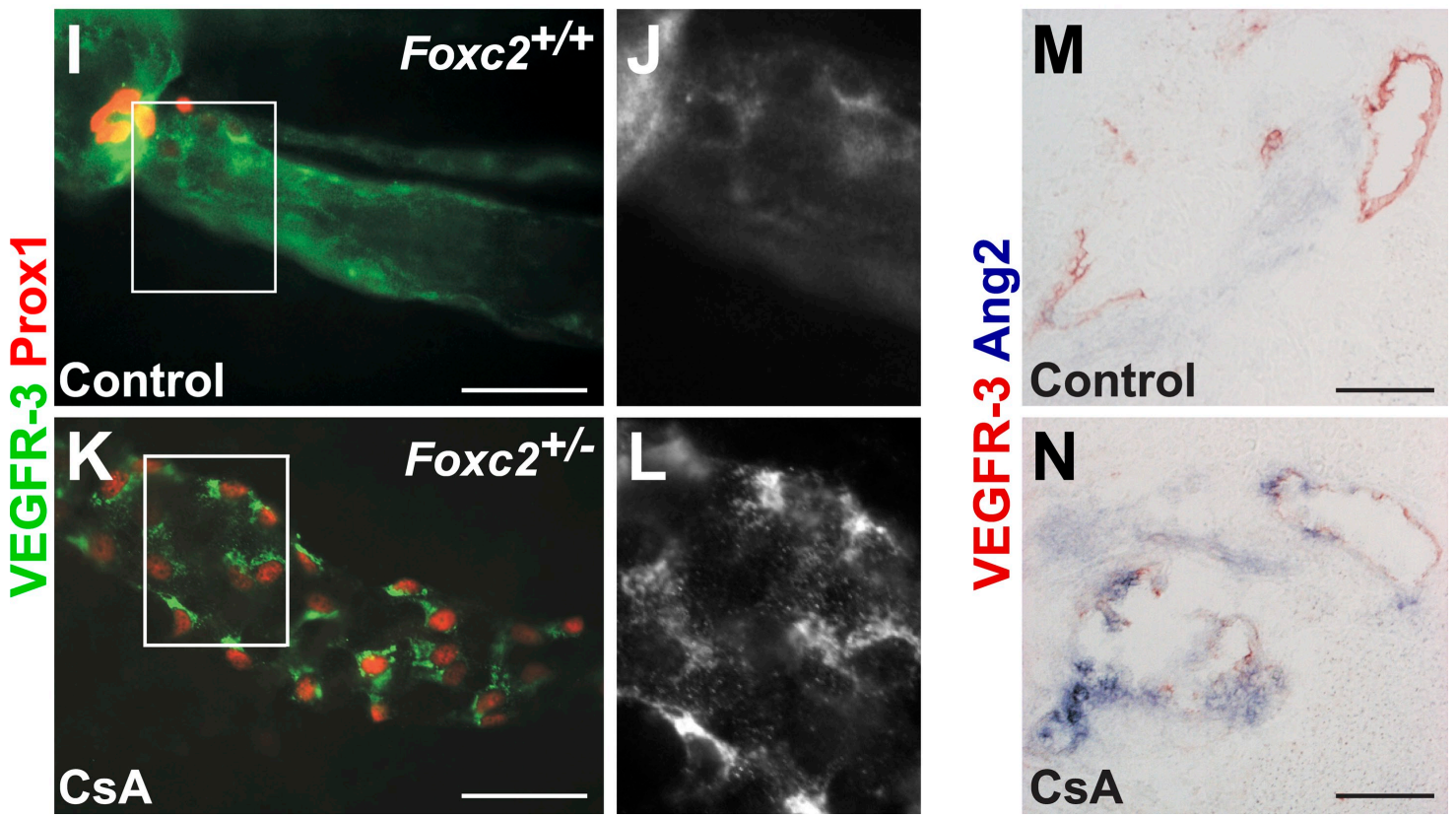

Figure 8. Inhibition of NFAT signaling in vivo prevents maturation of collecting lymphatic vessels. (A-H) Ectopic SMCs in lymphatic capillaries of Foxc2 ${ }^{-1-}$ and CsA-treated embryos. Whole-mount immunofluorescent staining for SMA (red) and Proxl (green) of embryonic skin at E17.5. Arrows, SMA-positive lymphatic vessels. (I-L) Increased VEGFR-3 internalization in lymphatic vessels of CsA-treated Foxc2 ${ }^{+/-}$embryos. Whole-mount immunofluorescent staining for VEGFR-3 (green/gray) and Prox1 (red). (M and N) Increased expression of Angpt2 upon inhibition of NFAT signaling. In situ hybridization for Angpt2 (blue) and immunohistochemical staining for VEGFR-3 (red) at E17.5. Boxed areas indicate regions shown at higher magnifications in J and L. Bars: (A-L) $100 \mu \mathrm{m} ;(\mathrm{M}$ and N) $25 \mu \mathrm{m}$.

understanding of the FOXC2-mediated transcriptional network, we performed genome-wide location analysis of endogenous FOXC2 in primary LECs. We used chromatin immunoprecipitation (ChIP) coupled with tiling microarrays covering the entire human genome (ChIP-chip) to produce an unbiased high resolution map of FOXC2-binding sites. For each ChIP-chip biological replicate, the immunoprecipitation was verified by Western blotting with anti-FOXC2 antibody (Fig. S4 A). 
A

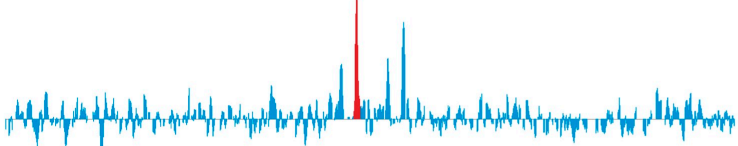

| | | |

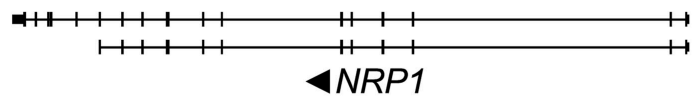

$33,520,000 \quad 33,580,000 \quad 33,640,000$

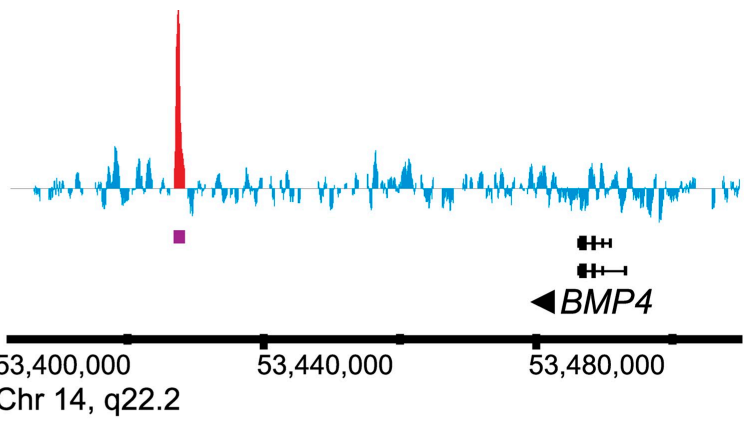

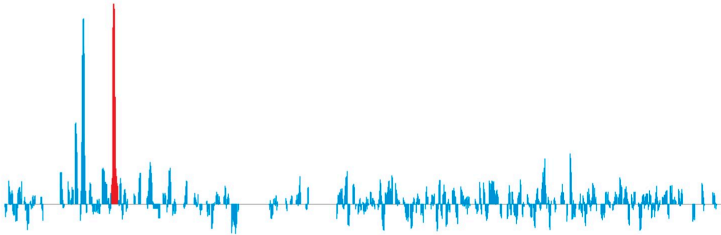

11

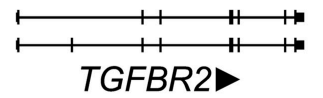

$30,520,000 \quad 30,600,000 \quad 30,680,000$

Chr 3, p24.1

B
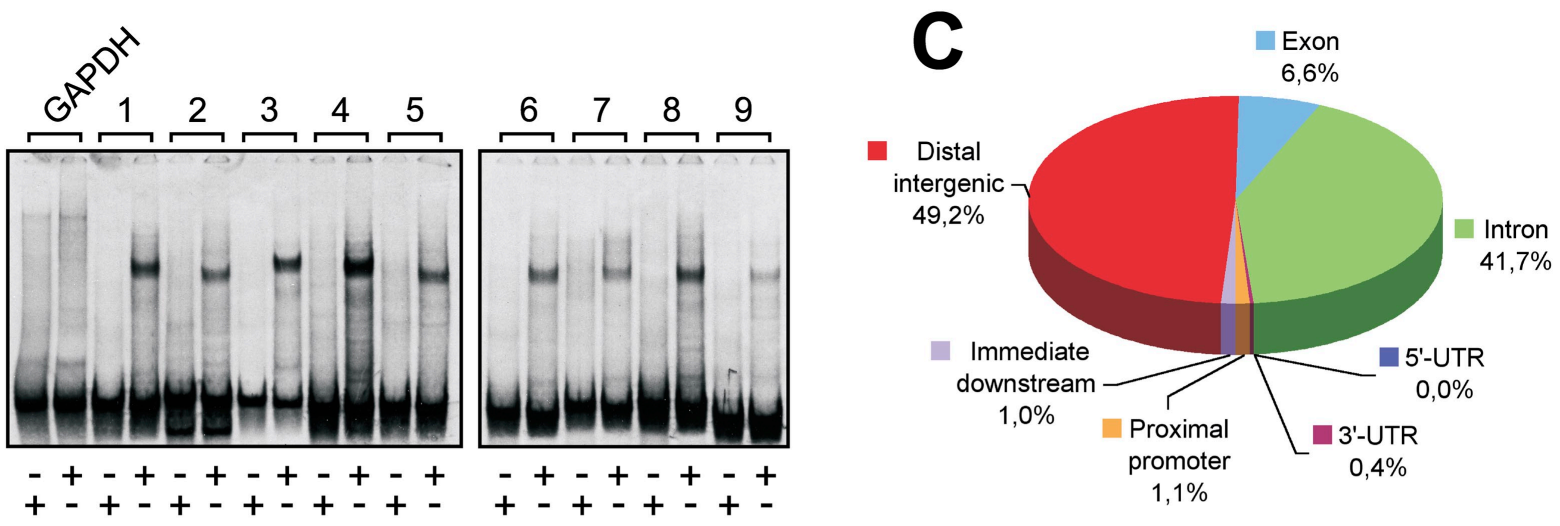

AdFOXC2

AdLacZ + - + - + - + - + + - + - + - + + -
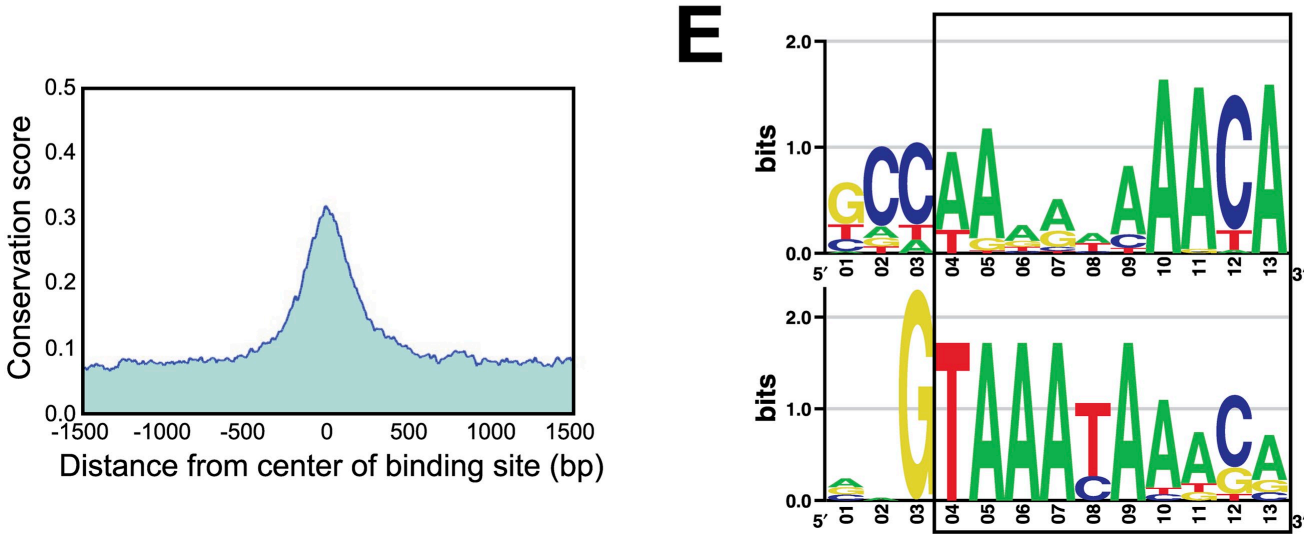

FOXC2

de novo discovered motif (ChIP-chip)

Freac-3 (FOXC1)

TRANSFAC M00291 (in vitro PCR selection)

Figure 9. Location analysis of FOXC2-binding sites in LECs. (A) Visualization of several FOXC2-binding regions. MAT score for each tiling array probe is shown in blue. Purple bars indicate FOXC2-enriched regions. Red peaks indicate ChIP regions selected for validation by EMSA. Potential target genes are indicated below the enrichment profile. Vertical lines, exons; horizontal lines, introns. Arrowheads indicate the direction of transcription. (B) Validation of 
ChIP DNA was amplified using the whole genome amplification method, which introduces very little bias compared with a commonly used linker-mediated PCR procedure (O'Geen et al., 2006). A total of 2350 FOXC2-binding sites were identified in LECs at a false discovery rate (FDR) of $0.1 \%$ (Fig. 9 and supplemental data). Because low yields of ChIP DNA from primary LECs precluded large-scale validation of ChIP-chip results by quantitative PCR, we used an alternative validation strategy by electrophoretic mobility shift assay (EMSA). We PCR amplified 200-bp fragments from nine randomly selected ChIP peak regions (Fig. S4 D) and tested them in EMSA with nuclear extracts from LECs transduced with FOXC2- (AdFOXC2) or control $\beta$-galactosidase-expressing adenoviruses (AdLacZ). The selection of ChIP regions was unbiased with respect to model-based analysis of tiling array (MAT) score, p-value, FDR, or fold change. As expected, FOXC2 formed stable complexes with all ChIP region fragments but not with the control glyceraldehyde 3-phosphate dehydrogenase (GAPDH) fragment, and no specific complexes were observed with control nuclear extracts from cells transduced with AdLacZ (Fig. 9 B). The FOXC2-DNA complexes could be supershifted with anti-FOXC2 but not control antibodies (Fig. S4 B; unpublished data), and point mutations in the forkhead-binding motif totally abolished the probe interaction with FOXC2 (Fig. S4 C).

Analysis of ChIP-chip data revealed that $>90 \%$ of FOXC2binding sites were located in distal intergenic regions and gene introns, and $<1 \%$ of sites were located in the proximal promoter regions (Fig. 9 C). Notably, there was a high degree of conservation of FOXC2-binding sites among higher eukaryotes (Fig. 9 D), suggesting that the FOXC2-regulated transcriptional network in lymphatic vascular development is conserved across species.

De novo motif discovery analysis revealed a common sequence motif in ChIP regions, which strongly resembled that of Freac-3 (FOXC1) from the TRANSFAC database. Both the newly discovered motif and the Freac- 3 motif contained the 3 '-terminal forkhead-binding element (T/A/C)AAACA preceded by an adenine-rich tract (Fig. 9 E). Such similarity between the two motifs is in good agreement with the fact that Freac-3/FOXC1 and FOXC2 have nearly identical DNA-binding domains (Saleem et al., 2004). The motifs, however, were completely different further upstream, which likely reflects fundamentally different approaches used for their identification. The Freac-3 motif has been identified by in vitro PCR selection using GST-tagged DNAbinding domain of FOXC1 (Pierrou et al., 1994), whereas the motif discovered in this study was derived from ChIP-chip experiments performed with endogenous FOXC2 in primary cells.
We further studied whether the ChIP-chip data could suggest an explanation for the observed physical interaction of FOXC2 and NFATc1 as well as for their functional cooperation in the in vivo models. We performed enrichment analysis of all human transcription factor-binding sites from the TRANSFAC database in the FOXC2 ChIP sequences. NFAT-binding sites were significantly enriched (M00302, 2.07 fold change and 95\% confidence interval [1.96; 2.19]; M00935, 1.95 fold change [1.84; 2.07]), whereas no statistically significant enrichment was seen for any of the control transcription factors (Fig. 10 A). Similarly, no enrichment was observed for transcription factors specifically expressed in endothelial cells (GATA2; Khandekar et al., 2007) or in LECs (SOX18, MEF2C, or PROX1; Wigle and Oliver, 1999; Wick et al., 2007; Francois et al., 2008; unpublished data). $86 \%$ of the FOXC2 ChIP sequences were found to contain at least one NFAT consensus sequence using a threshold of 3.4, which detects FOXC2-binding sites in $80 \%$ of the ChIP sequences (Table S1). Further experimental validation of the randomly selected $\sim 200$-bp fragments from FOXC2 ChIP peak regions (Fig. S4 D) by EMSA revealed that NFATc1 strongly interacted with fragments $3,4,7$, and 8 but not with fragments 1,2 , 5,6 , and 9. This observation was in agreement with the presence of NFAT consensus sites in the fragments 3, 4, 7, and 8 (Fig. 10 B; unpublished data). We next tested the fragments in luciferase reporter analysis and found that fragment 8 showed synergistic activation in the presence of both NFATc1 and FOXC2 compared with weak activation when either transcription factor was present alone (Fig. $10 \mathrm{C}$ ). Interestingly, the NFATc1-dependent activation of the reporter fragment 3 was partially repressed by FOXC2, whereas activity of reporter fragments 1 and 2 was not modified. The absence of response to FOXC2 of the reporters 1 and 2 could be a result of the fact that some 200-bp ChIP fragments may lack control elements needed for the efficient transcriptional regulation in our reporter system, or, alternatively, only the fragments that harbor both NFAT- and FOXC2-binding sites are capable of directing transcription. To investigate the mechanisms of transcriptional regulation by FOXC2 and NFATc1 in more detail, we performed site-directed mutagenesis of the reporter fragment 8 , which contained three potential FOXC2-binding sites and one NFAT-binding site. Inactivation of FOXC2 site 1 proximal to the NFAT site significantly reduced the response to NFATc1, whereas mutations of more distal FOXC2 sites 2 and 3 had less pronounced effects (Fig. 10 D). These results suggest that FOXC2 binding is required for further reporter activation by NFATc1.

In summary, we have identified FOXC2-binding sites in primary LECs using an unbiased genome-wide location mapping approach and showed that a subset of these regions is enriched

ChIP-chip results. ${ }^{33}$ P-labeled 200-bp DNA probes from selected ChIP regions (Fig. S4 D) were incubated with nuclear extracts from LECs transduced with FOXC2-expressing (AdFOXC2) or control adenovirus (AdLacZ), and the resulting DNA-protein complexes were analyzed by EMSA. GAPDH probe was used as a negative control. (C) Distribution of FOXC2-binding sites in the human genome. ChIP regions were mapped to their nearest RefSeq genes using CEAS (Ji et al., 2006). Proximal promoters and immediate downstream regions were defined as $\leq 1 \mathrm{~kb}$ upstream from the transcription start site or $\leq 1 \mathrm{~kb}$ downstream from the $3^{\prime}$ end of a gene, respectively. Distal intergenic refers to all locations outside the boundaries of a gene and the $1 \mathrm{~kb}$ flanking the gene on either end. UTR, untranslated region. (D) FOXC2-binding regions are conserved among higher eukaryotes. ChIP regions were uniformly expanded to $3 \mathrm{~kb}$, aligned at the center, and the mean phastCons conservation score (Siepel et al., 2005) was retrieved from the University of California Santa Cruz genome resource (http://genome.ucsc.edu) for each aligned position. The mean phastCons score was plotted against the distance from ChIP region center (1.5 kb upstream and downstream). (E) Comparison of a novel FOXC2-binding motif identified by MEME analysis of ChIP-chip data with the Freac-3 (FOXC1) motif from the TRANSFAC database. The region of similarity is boxed. Chr, chromosome. 

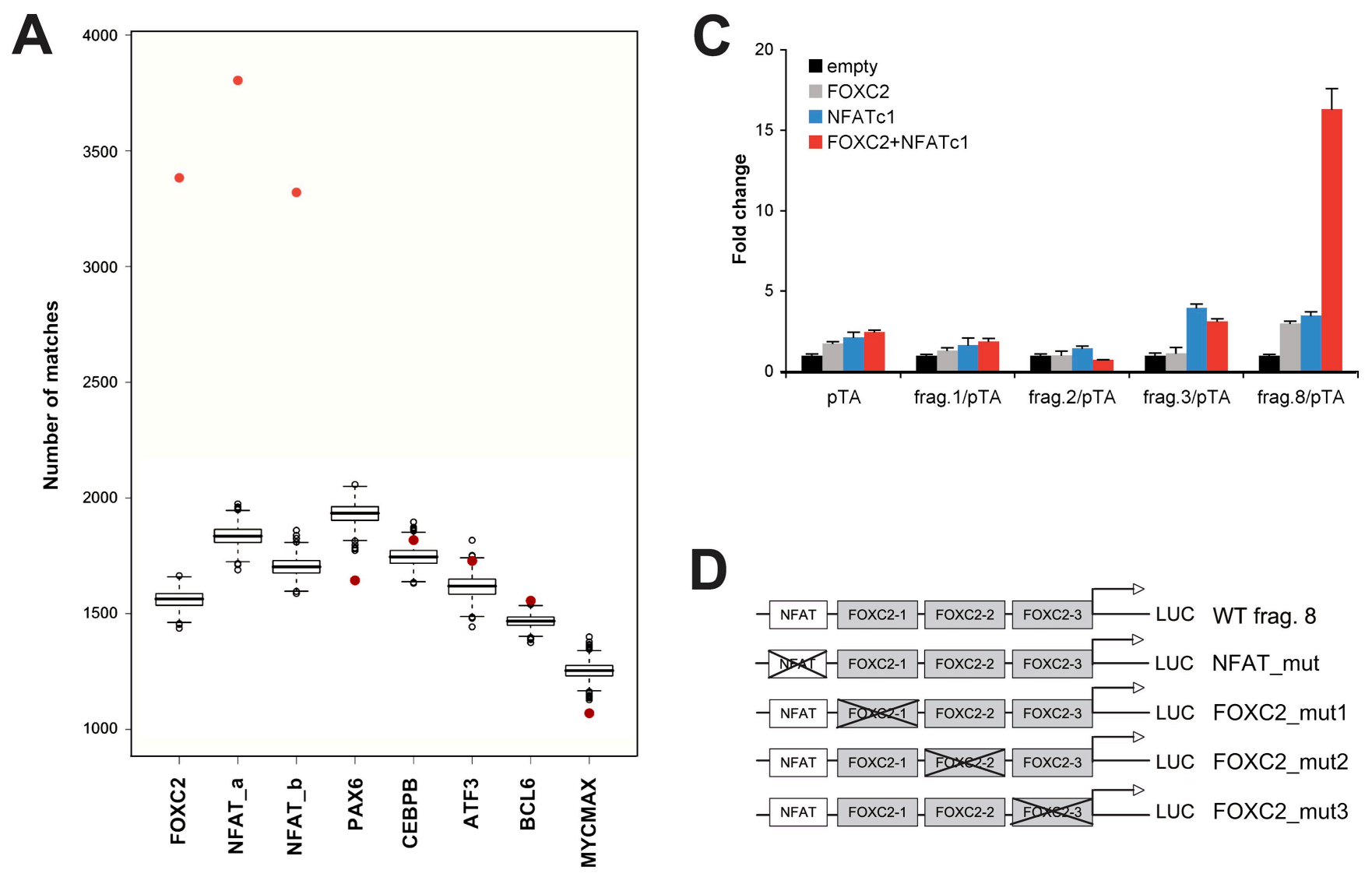

B
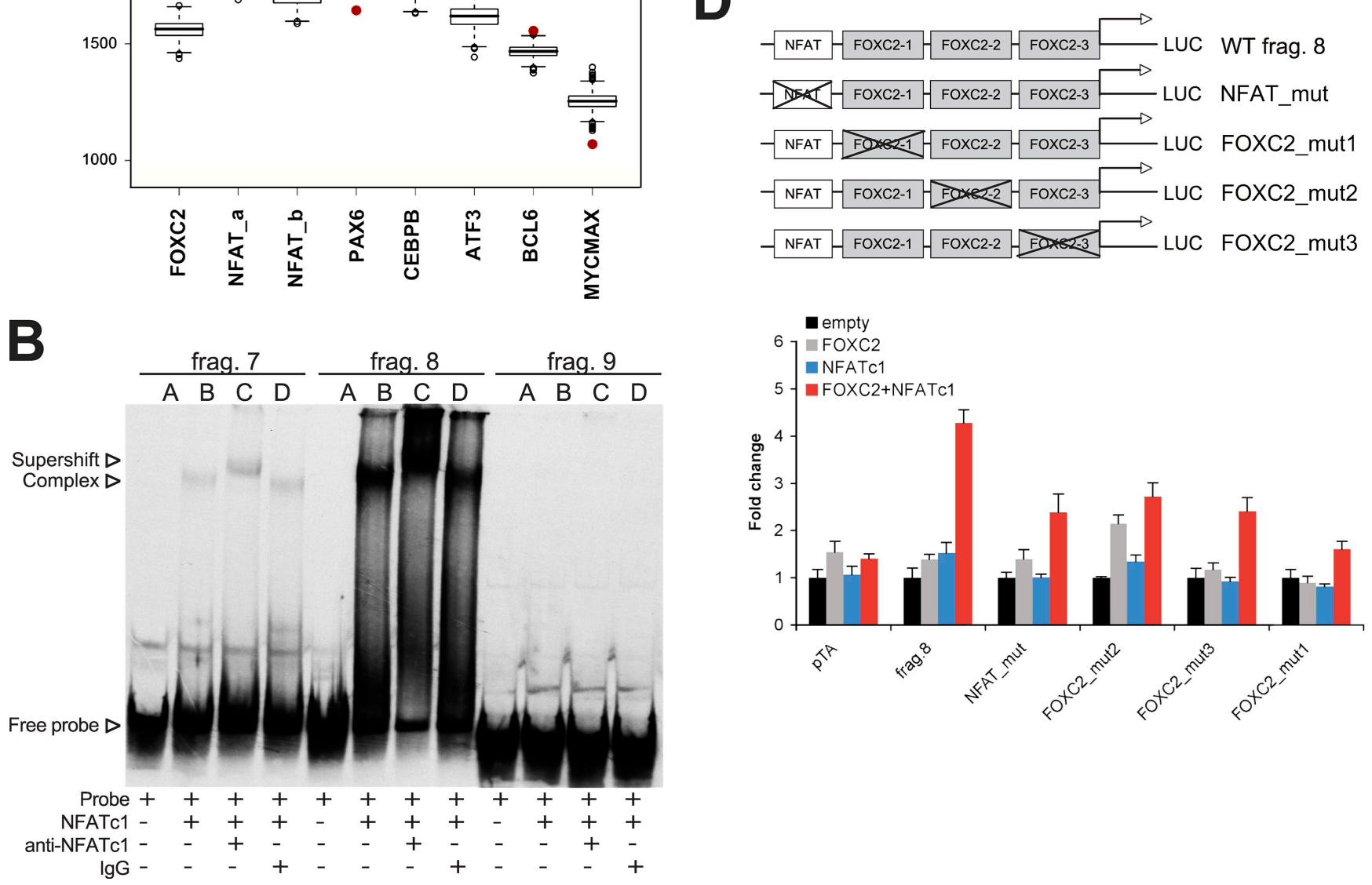

Figure 10. Cooperation of FOXC2 and NFATc1 signaling. (A) Enrichment of FOXC2- and NFATc1-binding sites in ChIP sequences. Red points indicate the number of sites observed in the original ChIP sequences, showing that both FOXC2 (de novo-discovered matrix)- and NFAT (M00302 and M00935)binding sites are highly enriched compared with the randomized sequences. PAX6 (M0097), CEBPB (M00109), ATF3 (M00513), BCL6 (M01183), and MYCMAX (M00322) are shown as examples of nonenriched sites. (B) NFATc1 interacts with a subset of FOXC2 ChIP regions. ${ }^{33}$ P-labeled DNA probes of $\sim 200$ bp from selected ChIP peak regions (Fig. S4 D) were incubated with recombinant NFATcl protein together with anti-NFATcl or control antibodies, and the resulting DNA-protein complexes were analyzed by EMSA. (C) Coregulation by FOXC2 and NFATc1. Selected $\sim 200-b p$ fragments of ChIP peak regions (Fig. S4 D) were subcloned into a pTA-luc luciferase reporter, and the luciferase activity was analyzed after cotransfection with either empty expression vector, expression vectors for FOXC2 or NFATc1, or both FOXC2 and NFATc1. (D) Mutations in FOXC2-binding sites reduce coactivation by NFATcl. Luciferase activity was analyzed after cotransfection of wild-type (WT) or mutated fragment 8/pTA-luc luciferase reporters with either empty expression vector, expression vectors for FOXC2 or NFATc1, or cotransfection with both FOXC2 and NFATc1. Data are presented as the mean \pm SD.

in NFAT-binding sites. In addition, we demonstrated that NFATc1 and FOXC2 interact with ChIP-derived DNA and that these two transcription factors can cooperate in the regulation of tran- scription. Collectively, these data provide a molecular basis for the observed in vivo cooperation between the NFATc1 and FOXC2 signaling in the developing lymphatic vasculature. 


\section{Discussion}

Blood vessel maturation, which starts with establishment of the venous-arterial identity and continues with the remodeling by sprouting, splitting, fusion, and pruning of preexisting vessels, recruitment of mural cells, and the deposition of a basement membrane and a perivascular ECM, has been extensively studied over recent years (Risau, 1997; Jain, 2003; Sato, 2003; Davis and Senger, 2005; Chantrain et al., 2006; Lamont and Childs, 2006). However, much less is known about the corresponding developmental process in the lymphatic vasculature. In this study, we describe the morphological and molecular changes that lead to the formation of the collecting lymphatic vessels during development. We conclude that the transcription factors FOXC2 and NFATc1 control this process and are responsible for establishing a collecting lymphatic vessel identity by binding to proximal sites on the DNA and regulating expression of downstream genes.

Discovery of lymphatic endothelial specific markers and molecular regulators, such as Prox1, VEGF-C-VEGFR-3, Foxc2, and LYVE-1, has significantly advanced our understanding of lymphatic vascular biology (Oliver, 2004; Makinen et al., 2007). In this study, we show that the specialization of lymphatic vessels into collecting vessels and capillaries is characterized by downregulation of lymphatic capillary markers LYVE-1, VEGFR-3, and SLC (Makinen et al., 2005; and this study), which is preceded by changes in the expression pattern of the transcription factors Foxc2 and Prox1. The role of LYVE-1 and SLC down-regulation in collecting lymphatic vessel function remains to be investigated. In contrast, down-regulation of VEGFR-3 is likely to be important for the establishment of a quiescent phenotype of collecting lymphatic vessels, which act essentially as lymph conduits, whereas lymphatic capillaries continue to express high levels of VEGFR-3 and serve as primary sites of lymphangiogenic response (Wirzenius et al., 2007). Such balance in response to VEGF-C signaling would also be important to ensure maximally efficient lymph transport. Accumulation of ECM and basement membrane components, as well as recruitment of pericytes and SMCs, is a relatively late event in the establishment of a collecting vessel identity, which follows the changes in gene expression in the lymphatic endothelium. Interestingly, VEGFR-3, Prox1, and Foxc2 levels remain high in the developing and adult intraluminal valves, suggesting that valve LECs are molecularly distinct from the nearby cells in the collecting vessel trunk, perhaps as a result of distinct mechanosensory transduction in these two regions and the need for the constant repopulation of endothelial cells in the valves.

Sharp up-regulation of Foxc2 at E15-15.5 is the earliest observed event during the formation of collecting vessels. Furthermore, genetic loss of Foxc2 leads to failure in the remodeling and the accompanying down-regulation of Prox1, VEGFR-3, LYVE-1, and SLC in maturing lymphatic vessels, which instead remain in an immature capillary-like state. Studies of the blood vasculature have demonstrated a functional separation of endothelial cells in angiogenic sprouts into stalk cells and tip cells, the latter characterized by extensive formation of filopodia, which is important for correct vessel guidance and establishment of a properly patterned vascular network in response to a VEGF gradient (Gerhardt et al., 2003). In this study, we show that in spite of excessive VEGFR-3 expression and lymphatic vessel hyperplasia, the Foxc2-deficient capillary plexus is paradoxically characterized by defective sprouting. Although the exact cause remains to be investigated, this is possibly a result of abnormal deposition of collagen IV around nascent tip cells, which could prevent formation of tip cell filopodia and normal vessel guidance and patterning. An important remaining question concerns the signals controlling Foxc2 expression in the developing lymphatic vasculature; studies from other cell types show that TGF- $\beta$ or BMP2 can induce FOXC2 in vitro (Yang et al., 2000; Mani et al., 2007). Future studies of mice with conditional ablation of TGF or bone morphogenetic proteins signaling components in the lymphatic endothelium should clarify the contribution of these pathways to Foxc2 regulation.

In search of similarities between the molecular mechanisms of cardiac and lymphatic valve formation, we found that NFATc1, a transcription factor that controls the morphogenesis of cardiac valves, is expressed in the developing lymphatic vasculature and intraluminal valves. Our data from primary LECs show that PROX1, at least in part, controls the LEC-specific expression of NFATc1, whereas the lymphangiogenic growth factor VEGF-C induces NFATc1 nuclear translocation through activation of VEGFR-2. The relative extent of the contribution of VEGFR-2-VEGFR-2 homodimeric versus VEGFR-2-VEGFR-3 heterodimeric complexes to NFATc1 activation remains to be determined; however, the inability of VEGFR-3 signaling alone to activate NFATc1 nuclear translocation suggests that in the lymphatic endothelium VEGFR-2- or VEGFR-2-VEGFR-3mediated pathways are more important for the establishment of collecting vessel phenotype rather than for the formation of a capillary network. In agreement with this hypothesis, VEGFR-2 is highly expressed in collecting lymphatic vessels and valves (Saaristo et al., 2002b; Wirzenius et al., 2007; unpublished data) where it promotes lymphatic vessel enlargement, whereas VEGFR-3 signaling is responsible for capillary sprouting (Wirzenius et al., 2007).

In addition to its role in endocardial cells, NFAT signaling in the blood vascular endothelium is important for cell proliferation, expression of proinflammatory genes, vascular patterning during early embryogenesis, and angiogenesis under pathological conditions (Schulz and Yutzey, 2004; for review see Crabtree and Olson, 2002). Our in vivo data, from Nfatcl-deficient mice and after inhibition of NFAT activation by CsA, show that the calcineurin/NFAT pathway does not contribute to angiogenesis at late stages of embryonic development, nor does it affect LEC commitment. Instead, loss of NFAT signaling impairs the maturation and remodeling of the lymphatic vasculature as indicated by persistent expression of lymphatic capillary markers, lymphatic vessel hyperplasia, reduced formation of lymphatic valves, and impaired sprouting. These phenotypes, reminiscent of the ones observed in Foxc $2^{-1-}$ mice, are strongly enhanced upon loss of one Foxc 2 allele, suggesting a genetic interaction between these two pathways. However, in CsA-treated mice, Foxc2 expression is high in all LECs, whereas NFATc1 expression is normal in Foxc2-deficient mice. These results indicate that Foxc 2 and NFATc1 are expressed independently of each other 
and suggest that they act by coregulating transcription of downstream genes.

To gain mechanistic understanding of the role of FOXC2 in LECs, we performed a genome-wide analysis of FOXC2binding sites. Previous studies suggested several FOXC2 target genes in blood vascular endothelium based on computational analysis of proximal promoters (Hayashi and Kume, 2008a,b; Hayashi et al., 2008). In this report, we have studied the association of FOXC2 across the entire genome, and we show that the vast majority of FOXC2-binding sites are located in the intergenic and intronic regions, arguing that FOXC2 acts primarily via distal enhancers as previously observed for estrogen-related receptor $\alpha$ and FOXa1 (Carroll et al., 2005; Lupien et al., 2008). The analysis of FOXC2 ChIP sequences demonstrated that NFAT-binding sites are strongly enriched in the proximity of FOXC2-binding sites. Based on this observation, we experimentally confirmed that NFATc1 binds to FOXC2 ChIP-derived fragments containing NFAT consensus and that it can direct transcription in reporter assays, thus providing the molecular basis for the NFAT and FOXC2 cooperation in vivo. The outstanding remaining question concerns the identification of FOXC2/NFATc1 effector genes or pathways responsible for establishment of the lymphatic collecting vessel phenotype. Future studies, including the combination of ChIP-chip data with chromosome capture analysis, gene expression profiling of Foxc2-deficient LECs, and large-scale screening studies in small vertebrate models of lymphatic vascular development, such as zebrafish or Xenopus laevis, will be invaluable to answer these questions.

In conclusion, our data provide a new understanding of the developmental regulation of lymphatic vessels and the role of Foxc2 in establishing a collecting vessel identity and introduce a novel role for the transcription factor NFATc1 in lymphatic development. Based on our findings, we propose a model for the role of Foxc2 and NFATc1 in establishing the identity of collecting lymphatic vessels. During normal lymphatic development, maturing lymphatic vessels up-regulate Foxc2, and VEGF-C-VEGFR-2/3 signaling activates NFATc1, which together start a genetic program for formation of collecting vessels. Deficiency in either Foxc2 or active NFATc1 in the lymphatic endothelium affects the expression of target genes, shutting down the program for establishment of a collecting vessel identity and leading to a persistent capillary-like phenotype. As damage to collecting vessels is a leading cause of secondary lymphedema, which frequently develops after cancer surgery-associated lymph node dissection, our data suggest that therapeutic delivery of FOXC2 and constitutively active NFATc1 could represent a strategy for treatment of secondary lymphedema through enhanced regeneration of collecting lymphatic vessels.

\section{Materials and methods}

\section{Animal models and in vivo analyses}

Animal experiments were approved by the National Animal Ethics Committee and were performed in accordance with institutional guidelines. Foxc2 $2^{-1-}$ mice were bred on the ICR background and genotyped as described previously (lida et al., 1997; Petrova et al., 2004). NFATc 1 ${ }^{-1-}$, Angpt2+/lacZ, TOP-Gal mice, and BAT-Gal mice have been described previously (Ranger et al., 1998; DasGupta and Fuchs, 1999; Gale et al., 2002; Maretto et al., 2003). For experiments involving only wild-type mice, outbred ICR or NMRI mice were used. Embryonic age was determined according to the day of the vaginal plug (E0.5). For NFAT inhibition, E12.5-16.5 pregnant mice were given $50 \mathrm{mg} / \mathrm{kg}$ CsA (EMD) in 10\% DMSO in sunflower seed oil (SigmaAldrich) or DMSO in oil only twice a day by oral gavage.

For fluorescent lymphangiography, embryos were injected with $10 \mathrm{mg} / \mathrm{ml}$ FITC-dextran in PBS (2,000-kD molecular mass; Sigma-Aldrich) subcutaneously in the hind limbs immediately after dissection, and flank collecting lymphatic vessels were visualized by fluorescence microscopy.

\section{Antibodies, staining procedures, and image acquisition}

Mesenteries and skins were dissected, fixed in 4\% PFA, and whole-mount staining was performed as described previously (Saaristo et al., 2002a; Tammela et al., 2005). 6- $\mu \mathrm{m}$ deparaffinized tissue sections were subjected to heat-induced epitope retrieval (High pH Retrieval Solution; Dako). Cultured cells were fixed with $4 \%$ PFA, permeabilized with $0.1 \%$ Triton X-100, and blocked with $5 \%$ donkey serum. For stainings, we used rat anti-mouse Foxc2 (Furumoto et al., 1999), mouse anti-human NFATcl (clone 7A6; Santa Cruz Biotechnology, Inc.; Timmerman et al., 1997), rabbit antihuman PROX1 (Karkkainen et al., 2004), goat anti-mouse VEGFR-3 (R\&D Systems), rabbit anti-mouse LYVE-1 (Petrova et al., 2004), rat anti-mouse PECAM-1 (BD), rabbit anti-mouse NG2 (Millipore), Cy3-conjugated mouse anti-human $\alpha$-SMA (Sigma-Aldrich), rabbit anti-mouse collagen IV (Cosmo Bio), and goat anti-mouse SLC (R\&D Systems). Alexa Fluor 488, 594, and 647 fluorochrome-conjugated secondary antibodies (Invitrogen) were used for signal detection. The tyramide signal amplification method (Tyramide Signal Amplification Indirect; PerkinElmer) was used for the detection of VEGFR-3 after in situ hybridization.

Samples were mounted with DAPI-containing Vectashield (Vector Laboratories) and analyzed at room temperature with a confocal microscope (LSM 510 Meta; Carl Zeiss, Inc.) using LSM AIM software or an epifluorescent microscope (Axioplan 2; Carl Zeiss, Inc.) equipped with a charge-coupled device camera (AxioCam HRc; Carl Zeiss, Inc.) using Axiovision software (version 4.6; Carl Zeiss, Inc.). Objectives used were 10x Plan-Apochromat NA 0.45, 10x Fluar NA 0.5, 10x Plan-Neofluar NA $0.3,20 \times$ Plan-Neofluar NA 0.5, 40x Plan-Apochromat NA 1.3, 40x longdistance C-Apochromat NA 1.1, and 40x Plan-Neofluar NA 0.75 (Carl Zeiss, Inc.). Three-dimensional projections of whole-mount images were digitally reconstructed from confocal z stacks using LSM AIM software and processed using Photoshop (CS; Adobe). Images are representative pictures from three to five embryos/stage or phenotype.

\section{In situ hybridization}

Frozen sections or whole-mount mesenteries from E17.5 embryos were used and processed as described previously (Wilkinson, 1998). Sections were hybridized with complementary RNA anti-sense or sense probes for Angpt2 (nucleotides 45-738 from GenBank under accession no. NM 007426), NFATc 1 (nucleotides 1,012-1,519 from GenBank under accession no. NM_016791), or Prox 1 (nucleotides 276-1,208 from GenBank under accession no. NM_002763).

\section{Cell culture, transfections, transductions, and luciferase reporter assays}

Human iLECs were isolated as described previously (Haraldsen et al., 1995; Jahnsen et al., 1997) and cultured in endothelial cell growth medium (PromoCell). Lymphatic endothelial identity was confirmed with PROXI staining. For growth factor treatments, iLECs were starved for $6 \mathrm{~h}$, treated with $200 \mathrm{ng} / \mathrm{ml} \mathrm{CsA} \mathrm{(EMD)} \mathrm{in} \mathrm{DMSO} \mathrm{or} \mathrm{only} \mathrm{DMSO} \mathrm{for} 2 \mathrm{~h}$ in starvation medium, and stimulated with $200 \mathrm{ng} / \mathrm{ml} \Delta \mathrm{N} \Delta C$ VEGF-C (Karpanen et al., 2006), $500 \mathrm{ng} / \mathrm{ml} \Delta \mathrm{N} \Delta$ C VEGF-C(C156S) (Heckman et al., 2008), and $500 \mathrm{ng} / \mathrm{ml}$ VEGF-E NZ7 (Gerhardt et al., 2003) for $20 \mathrm{~min}$. For siRNA experiments, iLECs were transfected with 10-40 nM siRNA using Lipofectamine 2000 (Invitrogen) and the following siRNAs: PROX1, 5'-CUAUGAGCCAGUUUGAUAUUU-3' (Thermo Fisher Scientific); FOXC2, 5'-AGGTGGTGATCAAGAGCGAUU-3'; or siRNA Allstars Control (Invitrogen). Cells were lysed 48-96 h after transfection. For adenoviral experiments, iLECs were transduced with 20-50 plaque-forming unit/cell recombinant adenoviruses encoding human PROX1 (Petrova et al., 2002), FOXC2 (Petrova et al., 2004), or LacZ (Laitinen et al., 1998) in serum-free medium for $1 \mathrm{~h}$ and lysed $48 \mathrm{~h}$ after transfection. For luciferase reporter assays, 183-199-bp sequences from selected ChIP peak regions were subcloned into pTA luciferase reporter vector. 293T cells were cotransfected with reporter constructs and constitutively active NFATc 1 (plasmid 11102; Addgene; Monticelli and Rao, 2002), FOXC2 (Petrova et al., 2004), and empty vectors using jetPEI transfection reagent (Polyplus-Transfection). 
Renilla luciferase pCMV-RL (provided by C. Heckman, University of Helsinki, Helsinki, Finland) was used for normalization of transfection efficiency. $48 \mathrm{~h}$ after transfection, cells were lysed and analyzed using Dual-Luciferase kit (Promega). Mutations in FOXC2-binding sites in fragment 8 were introduced using site-directed mutagenesis with the following primers: NFAT mutant, 5'-CAAAAACACACTCCAACCTTTCC-3' and 5'-GATGTAGGTCTAAGGGCGAATTC-3'; FOXC2 mutant 1, 5'-GACAGAAACCTATTATTGGAAACAATT-3' and 5'-CGTTCAGGGTAGGAGAAGGGAAG-3'; FOXC2 mutant 2, 5'-GACAGGATTCATATATTTCATCC-3' and 5'-CATAGACGTGTATGAAATAATTGTT-3'; and FOXC2 mutant 3, 5'-GACATGTCAAAATAGGAAGCCCA-3' and '5'-CTTATTTCCTGGATGAAATATATGAATCCTGT-3'. The presence of mutations was verified by sequencing. Experiments were done in triplicate and repeated at least three times. Values are expressed as fold increase compared with empty control \pm SD.

\section{ChIP-chip analysis}

ChIP-chip was performed essentially as described by O'Geen et al., (2006) with the following modifications: iLECs were cross-linked with formaldehyde in complete endothelial cell basal medium MV (PromoCell), and IP dilution buffer was substituted with $3 \%$ BSA containing complete inhibitor tablets (Roche). DNA was sheared using a sonicator (Soniprep 150; MSE) and immunoprecipitated using rat anti-mouse Foxc2 antibody (Furumoto et al., 1999) complexed with protein G-Sepharose (GE Healthcare). Decrosslinked ChIP DNA was amplified using GenomePlex Complete Whole Genome Amplification kit (Sigma-Aldrich) using the buffer supplemented with deoxynucleotide-triphosphate + deoxy-UTP mixture. DNA was fragmented using uracil DNA glycosylase and apurinic/apyrimidinic endonuclease. Terminal labeling, hybridization, and array processing were performed as recommended by the array manufacturer (Affymetrix). Three biologically independent ChIP replicates and two control lgG replicates were hybridized to GeneChip Human Tiling 2.0R Array Sets (Affymetrix). Data analysis was performed using MAT software (Johnson et al., 2006) at $0.1 \%$ FDR cutoff (intensity analysis parameters: BandWidth $=300$, MaxGap $=$ 300 , MinProbe $=10$ ). A total of 2,359 FOXC2-enriched regions were identified of which nine mitochondrial sequences were discarded from further analysis. Enriched regions were visualized using Integrated Genome Browser software (Affymetrix). Initial analyses of evolutionary conservation, nearby genes, and enriched transcription factor-binding motifs were performed using cis-regulatory element annotation system (CEAS) software (Ji et al., 2006).

\section{Bioinformatics analyses of ChIP-chip data}

400-bp-long fragments from the center of each ChIP sequence were extracted and ranked according to the p-value. The fragments were split in 10 bins of 235 sequences each, with bin 1 containing the sequences with the lowest p-values and bin 10 containing the sequences with the highest p-values. De novo motif discovery was performed in each bin separately using the program MEME (multiple EM for motif elicitation; Bailey and Elkan, 1994) in the mode of one occurrence per sequence and a motif width ranging from 7 to 15. MEME results were confirmed by the Gibbs sampler (Thompson et al., 2003) with default options.

The de novo-discovered FOXC2-binding site and all vertebrate transcription factor-binding sites stored as TRANSFAC matrices (Matys et al., 2003) were turned into calibrated profiles using the pftools program (Bucher et al., 1996; Roulet et al., 2002). To select a stringency threshold defining binding site predictions of high quality, several score thresholds were applied to detect potential FOXC2-binding sites in the 2,350 ChIP sequences, and the threshold of 3.4 was selected, as it predicted a FOXC2binding site in $80 \%$ of the sequences. This same threshold was then used to predict the positions of other transcription factor-binding sites such as NFAT. The NFAT matrix 1 has been derived from 26 experimentally identified endogenous NFAT-binding sites (Kel et al., 1999), and the NFAT matrix 2 has been derived from a selection of the same sites used for matrix 1 plus eight additional TRANSCompel sites. The same 3.4 cutoff was used to compute random matches in 1,000 random sets, which were composed of randomly shuffled ChIP sequences with a window of 20 nucleotides. The enrichment, or fold change, was computed as the ratio of the number of ChIP matches and the mean number of random matches. The $95 \%$ confidence interval for the enrichment ratios was estimated assuming that the sampling distribution is approximately Gaussian for the log ratio and Poisson for the counts. For the analysis of putative PROX1-binding sites, the matrix for Drosophila melanogaster prospero was used (Choksi et al., 2006).

\section{EMSA}

$25 \mu$ EMSA reaction mixtures contained $10 \mathrm{mM}$ Hepes, $\mathrm{pH}$ 8.0, $3 \mathrm{mM}$ Tris- $\mathrm{HCl}, \mathrm{pH} 8.0,50 \mathrm{mM} \mathrm{KCl}, 2 \mathrm{mM} \mathrm{MgCl} 2,10 \%$ glycerol, 1 mM dithiotreitol,
$0.04 \mathrm{mg} / \mathrm{ml}$ poly-deoxy-inosinic-deoxy-cytidylic acid, $0.4 \mathrm{mg} / \mathrm{ml} \mathrm{BSA}$, $\gamma^{33} \mathrm{P}$-labeled DNA probe, and cell lysates or $0.8 \mu \mathrm{g}$ recombinant human NFATcl protein (SignalChem). For supershifts, $1 \mathrm{\mu g}$ rat anti-mouse Foxc2, mouse anti-human NFATcl, or control antibodies from the same species were added to the reaction mixtures. After incubation for $30 \mathrm{~min}$ at room temperature, probes were loaded on $5 \%$ criterion Tris-Borate-EDTA polyacrylamide gel (Bio-Rad Laboratories). Electrophoresis was performed for $1-2 \mathrm{~h}$ at $10-20 \mathrm{~mA} / 100-150 \mathrm{~V}$. The gels were dried and visualized by autoradiography or using a phosphorimager.

\section{Immunoprecipitation and Western and Northern blotting}

For analysis of protein interaction, 293T cells were cotransfected with HANFATc 1 and FOXC2 expression vectors using Lipofectamine 2000 (Invitrogen). $48 \mathrm{~h}$ after transfection, cells were lysed with buffer containing $20 \mathrm{mM}$ Tris, pH 7.8, $500 \mathrm{nM} \mathrm{NaCl}, 1 \% \mathrm{NP}-40$, and complete protease inhibitor mixture (Roche). The lysates were sonicated, spinned down, precleared with protein G-Sepharose beads (GE Healthcare), incubated overnight with anti-HA antibodies (Abcam) or control nonimmune rabbit lgG (SigmaAldrich), and incubated with protein G-Sepharose beads. The precipitates were washed with lysis buffer, resuspended in Laemmli sample buffer, and heated at $95^{\circ} \mathrm{C}$ for $10 \mathrm{~min}$.

For Western blotting, samples were resolved by SDS-PAGE, transferred onto Immobilon-P membrane (Millipore), and blotted with monoclonal antibodies against Foxc2 (Furumoto et al., 1999), NFATc1 (clone 7A6; Santa Cruz Biotechnology, Inc.), PROX I (R\&D Systems), phospho-VEGFR-2 (Tyr1 175; Cell Signaling Technology), phospho-ERK1/2 (Cell Signaling Technology), and GAPDH (Europa Bioproducts). Western blots were developed using the ECL method (SuperSignal West Femto Maximum Sensitivity Substrate; Thermo Fisher Scientific).

For Northern analysis, $10 \mathrm{\mu g}$ total RNA was separated in a $1 \%$ agarose gel, and blots were hybridized in ULTRAhyb solution (Applied Biosystems) with a ${ }^{32} \mathrm{P}$-labeled fragment of human NFATCl (nucleotides 2,577-2,825 from GenBank under accession no. NM_006162).

\section{Quantification and statistical analysis}

Mesenteric valves were identified as areas of strongly positive Prox 1 staining and counted from duodenum to ileum ( $n=4$ for each genotype and treatment; E17.5). The mean vessel widths of dermal capillaries $(n=4)$ were calculated using Matlab (Mathworks) by dividing vessel area (determined by thresholding) by length (determined by a skeletonization algorithm by Nicholas R. Howe, Smith College, Northampton, MA). For colocalization of Foxc2 and NFATc1, LECs were counted from nine different lymphatic vessels from paraffin sections of three wild-type E17.5 embryos. For analysis of nuclear NFATcl intensity in LECs, RGB images were analyzed with the CellCounter program (developed by V. Rantanen, Molecular Imaging Unit, University of Helsinki). Values were counted from nuclear NFATcl staining and expressed as mean percentual intensity of maximum pixel intensity. A two-tailed, unpaired Student's $t$ test was performed to determine statistical significance by the probability of difference between the means.

\section{Online supplemental material}

Fig. S1 shows LEC clusters and filopodia in the developing mouse mesentery at E14.5 and heterogeneous expression of LYVE-1 in embryonic mesentery and skin during development. Fig. S2 shows analysis of NFATc 1 expression by in situ hybridization, colocalization with Foxc2, inhibition of NFATc 1 nuclear translocation, and Foxc2 expression in CsA-treated mice. Fig. S3 shows activation of ERK1/2 phosphorylation by wild-type and mutant growth factors, equal NFATcl mRNA levels in iLECs after growth factor stimulation, and an increase in overall NFATc 1 expression upon PROX1 overexpression, coimmunoprecipitation of FOXC2, and NFATc 1. Fig. S4 shows Western blots of FOXC2 ChIP experiments, binding of FOXC2 to ChIP targets in EMSA, which is abolished by mutations in FOXC2-binding sites, and a table with ChIP region fragments used in EMSAs and luciferase reporter assays. The supplemental data contains the CEAS annotation results of all FOXC2 ChIP sequences, whereas Table S1 shows FOXC2 ChIP regions that also were found to contain NFATbinding sites. Online supplemental material is available at http://www.jcb .org/cgi/content/full/jcb.200901104/DC1.

We thank Dr. Elaine Fuchs and Dr. Nicole Stokes (Howard Hughes Medical Institute, The Rockefeller University, New York, NY) for E15.5 NFATc 1-1embryos, Drs. Gerald Crabtree and Wen Qi Ho (Stanford University School of Medicine, Stanford, CAl for E12.5 NFATc 1 ${ }^{-1-}$ embryos, Dr. Stefano Piccolo (University of Padua, Padua, Italy) for BAT-Gal mice, Dr. Michael Jeltsch (University of Helsinkil for VEGF-C and VEGF-E proteins, Dr. Caroline Heckman 
(University of Helsinki) for the pCMV-RL luciferase plasmid, and Dr. Ernest Mueller from Sigma-Aldrich for the custom-formulated WGA buffer. We gratefully acknowledge Alun Parsons, Tapio Tainola, Sanna Wallin, Kaisa Makkonen, Sanna Lampi, Paula Hyvärinen, and Mari Helanterä for technical support, the Biomedicum Helsinki Molecular Imaging Unit and Ville Rantanen for technical assistance on imaging and image analysis, and Outi Monni and Hanna Pesonen for help in microarray processing. Microarray hybridization was carried out at Biomedicum Biochip Center.

The study was supported by the Academy of Sciences of Finland, Sigrid Juselius Foundation, Swiss National Science Foundation, Magnus Ehrnrooth Foundation, Louis-Jeantet Foundation, European Union (Lymphangiogenomics project no. LSHG-CT-2004-503573), K. Albin Johansson Foundation, Helsinki Biomedical Graduate School, Nylands Nation, Einar and Karin Stroem's Foundation, Svenska Kulturfonden, Biomedicum Helsinki Foundation, Medicinska Understödsföreningen Liv och Hälsa, Paulos Foundation, Orion-Farmos Research Foundation, and Deutsche Forschungsgemeinschaft (SFB-TR23 A3 Vascular Differentiation and Remodeling grant to H.G. Augustin)

Submitted: 21 January 2009

Accepted: 2 April 2009

\section{References}

Armstrong, E.J., and J. Bischoff. 2004. Heart valve development: endothelial cell signaling and differentiation. Circ. Res. 95:459-470.

Bailey, T.L., and C. Elkan. 1994. Fitting a mixture model by expectation maximization to discover motifs in biopolymers. Proc. Int. Conf. Intell. Syst. Mol. Biol. 2:28-36.

Bettelli, E., M. Dastrange, and M. Oukka. 2005. Foxp3 interacts with nuclear factor of activated T cells and NF-kappa B to repress cytokine gene expression and effector functions of T helper cells. Proc. Natl. Acad. Sci. USA. 102:5138-5143.

Brice, G., S. Mansour, R. Bell, J.R. Collin, A.H. Child, A.F. Brady, M. Sarfarazi, K.G. Burnand, S. Jeffery, P. Mortimer, and V.A. Murday. 2002. Analysis of the phenotypic abnormalities in lymphoedema-distichiasis syndrome in 74 patients with FOXC2 mutations or linkage to 16q24. J. Med. Genet. 39:478-483.

Bucher, P., K. Karplus, N. Moeri, and K. Hofmann. 1996. A flexible motif search technique based on generalized profiles. Comput. Chem. 20:3-23.

Burri, P.H., R. Hlushchuk, and V. Djonov. 2004. Intussusceptive angiogenesis: its emergence, its characteristics, and its significance. Dev. Dyn. 231:474-488.

Carroll, J.S., X.S. Liu, A.S. Brodsky, W. Li, C.A. Meyer, A.J. Szary, J. Eeckhoute, W. Shao, E.V. Hestermann, T.R. Geistlinger, et al. 2005. Chromosomewide mapping of estrogen receptor binding reveals long-range regulation requiring the forkhead protein FoxA1. Cell. 122:33-43.

Chang, C.P., J.R. Neilson, J.H. Bayle, J.E. Gestwicki, A. Kuo, K. Stankunas, I.A. Graef, and G.R. Crabtree. 2004. A field of myocardial-endocardial NFAT signaling underlies heart valve morphogenesis. Cell. 118:649-663.

Chantrain, C.F., P. Henriet, S. Jodele, H. Emonard, O. Feron, P.J. Courtoy, Y.A. DeClerck, and E. Marbaix. 2006. Mechanisms of pericyte recruitment in tumour angiogenesis: a new role for metalloproteinases. Eur. J. Cancer. 42:310-318.

Choksi, S.P., T.D. Southall, T. Bossing, K. Edoff, E. de Wit, B.E. Fischer, B. van Steensel, G. Micklem, and A.H. Brand. 2006. Prospero acts as a binary switch between self-renewal and differentiation in Drosophila neural stem cells. Dev. Cell. 11:775-789.

Crabtree, G.R., and E.N. Olson. 2002. NFAT signaling: choreographing the social lives of cells. Cell. 109(Suppl):S67-S79.

DasGupta, R., and E. Fuchs. 1999. Multiple roles for activated LEF/TCF transcription complexes during hair follicle development and differentiation. Development. 126:4557-4568.

Davis, G.E., and D.R. Senger. 2005. Endothelial extracellular matrix: biosynthesis, remodeling, and functions during vascular morphogenesis and neovessel stabilization. Circ. Res. 97:1093-1107.

de la Pompa, J.L., L.A. Timmerman, H. Takimoto, H. Yoshida, A.J. Elia, E. Samper, J. Potter, A. Wakeham, L. Marengere, B.L. Langille, et al. 1998. Role of the NF-ATc transcription factor in morphogenesis of cardiac valves and septum. Nature. 392:182-186.

Dellinger, M., R. Hunter, M. Bernas, N. Gale, G. Yancopoulos, R. Erickson, and M. Witte. 2008. Defective remodeling and maturation of the lymphatic vasculature in Angiopoietin-2 deficient mice. Dev. Biol. 319:309-320.

Dumont, D.J., L. Jussila, J. Taipale, A. Lymboussaki, T. Mustonen, K. Pajusola, M. Breitman, and K. Alitalo. 1998. Cardiovascular failure in mouse embryos deficient in VEGF receptor-3. Science. 282:946-949.
Francois, M., A. Caprini, B. Hosking, F. Orsenigo, D. Wilhelm, C. Browne, K. Paavonen, T. Karnezis, R. Shayan, M. Downes, et al. 2008. Sox 18 induces development of the lymphatic vasculature in mice. Nature. 456:643-647.

Furumoto, T.A., N. Miura, T. Akasaka, Y. Mizutani-Koseki, H. Sudo, K. Fukuda, M. Maekawa, S. Yuasa, Y. Fu, H. Moriya, et al. 1999. Notochord-dependent expression of MFH1 and PAX1 cooperates to maintain the proliferation of sclerotome cells during the vertebral column development. Dev. Biol. 210:15-29.

Gale, N.W., G. Thurston, S.F. Hackett, R. Renard, Q. Wang, J. McClain, C. Martin, C. Witte, M.H. Witte, D. Jackson, et al. 2002. Angiopoietin-2 is required for postnatal angiogenesis and lymphatic patterning, and only the latter role is rescued by Angiopoietin-1. Dev. Cell. 3:411-423.

Gerhardt, H., M. Golding, M. Fruttiger, C. Ruhrberg, A. Lundkvist, A. Abramsson, M. Jeltsch, C. Mitchell, K. Alitalo, D. Shima, and C. Betsholtz. 2003. VEGF guides angiogenic sprouting utilizing endothelial tip cell filopodia. J. Cell Biol. 161:1163-1177.

Graef, I.A., F. Chen, L. Chen, A. Kuo, and G.R. Crabtree. 2001. Signals transduced by $\mathrm{Ca}(2+) /$ calcineurin and NFATc3/c4 pattern the developing vasculature. Cell. 105:863-875.

Haraldsen, G., J. Rugtveit, D. Kvale, T. Scholz, W.A. Muller, T. Hovig, and P. Brandtzaeg. 1995. Isolation and longterm culture of human intestinal microvascular endothelial cells. Gut. 37:225-234.

Hayashi, H., and T. Kume. 2008a. Forkhead transcription factors regulate expression of the chemokine receptor CXCR4 in endothelial cells and CXCL12induced cell migration. Biochem. Biophys. Res. Commun. 367:584-589.

Hayashi, H., and T. Kume. 2008b. Foxc transcription factors directly regulate Dl14 and Hey2 expression by interacting with the VEGF-Notch signaling pathways in endothelial cells. PLOS ONE. 3:e2401.

Hayashi, H., H. Sano, S. Seo, and T. Kume. 2008. The Foxc2 transcription factor regulates angiogenesis via induction of integrin beta3 expression. J. Biol. Chem. 283:23791-23800.

Heckman, C.A., T. Holopainen, M. Wirzenius, S. Keskitalo, M. Jeltsch, S. YläHerttuala, S.R. Wedge, J.M. Jürgensmeier, and K. Alitalo. 2008. The tyrosine kinase inhibitor cediranib blocks ligand-induced VEGFR-3 activity and lymphangiogenesis. Cancer Res. 68:4754-4762.

Horsley, V., A.O. Aliprantis, L. Polak, L.H. Glimcher, and E. Fuchs. 2008. NFATc1 balances quiescence and proliferation of skin stem cells. Cell. 132:299-310.

Iida, K., H. Koseki, H. Kakinuma, N. Kato, Y. Mizutani-Koseki, H. Ohuchi, H. Yoshioka, S. Noji, K. Kawamura, Y. Kataoka, et al. 1997. Essential roles of the winged helix transcription factor MFH-1 in aortic arch patterning and skeletogenesis. Development. 124:4627-4638.

Jahnsen, F.L., P. Brandtzaeg, R. Haye, and G. Haraldsen. 1997. Expression of functional VCAM-1 by cultured nasal polyp-derived microvascular endothelium. Am. J. Pathol. 150:2113-2123.

Jain, R.K. 2003. Molecular regulation of vessel maturation. Nat. Med. 9:685-693.

Ji, X., W. Li, J. Song, L. Wei, and X.S. Liu. 2006. CEAS: cis-regulatory element annotation system. Nucleic Acids Res. 34:W551-W554.

Johnson, E.N., Y.M. Lee, T.L. Sander, E. Rabkin, F.J. Schoen, S. Kaushal, and J. Bischoff. 2003. NFATc1 mediates vascular endothelial growth factorinduced proliferation of human pulmonary valve endothelial cells. J. Biol. Chem. 278:1686-1692.

Johnson, W.E., W. Li, C.A. Meyer, R. Gottardo, J.S. Carroll, M. Brown, and X.S. Liu. 2006. Model-based analysis of tiling-arrays for ChIP-chip. Proc. Natl. Acad. Sci. USA. 103:12457-12462.

Joukov, V., V. Kumar, T. Sorsa, E. Arighi, H. Weich, O. Saksela, and K. Alitalo. 1998. A recombinant mutant vascular endothelial growth factor-C that has lost vascular endothelial growth factor receptor-2 binding, activation, and vascular permeability activities. J. Biol. Chem. 273:6599-6602.

Karkkainen, M.J., P. Haiko, K. Sainio, J. Partanen, J. Taipale, T.V. Petrova, M. Jeltsch, D.G. Jackson, M. Talikka, H. Rauvala, et al. 2004. Vascular endothelial growth factor $\mathrm{C}$ is required for sprouting of the first lymphatic vessels from embryonic veins. Nat. Immunol. 5:74-80.

Karpanen, T., C.A. Heckman, S. Keskitalo, M. Jeltsch, H. Ollila, G. Neufeld, L. Tamagnone, and K. Alitalo. 2006. Functional interaction of VEGF-C and VEGF-D with neuropilin receptors. FASEB J. 20:1462-1472.

Kel, A., O. Kel-Margoulis, V. Babenko, and E. Wingender. 1999. Recognition of NFATp/AP-1 composite elements within genes induced upon the activation of immune cells. J. Mol. Biol. 288:353-376.

Khandekar, M., W. Brandt, Y. Zhou, S. Dagenais, T.W. Glover, N. Suzuki, R. Shimizu, M. Yamamoto, K.C. Lim, and J.D. Engel. 2007. A Gata2 intronic enhancer confers its pan-endothelia-specific regulation. Development. 134:1703-1712.

Kinoshita, S., L. Su, M. Amano, L.A. Timmerman, H. Kaneshima, and G.P. Nolan. 1997. The T cell activation factor NF-ATc positively regulates HIV-1 replication and gene expression in T cells. Immunity. 6:235-244.

Kukk, E., A. Lymboussaki, S. Taira, A. Kaipainen, M. Jeltsch, V. Joukov, and K. Alitalo. 1996. VEGF-C receptor binding and pattern of expression 
with VEGFR-3 suggests a role in lymphatic vascular development. Development. 122:3829-3837.

Laitinen, M., K. Makinen, H. Manninen, P. Matsi, M. Kossila, R.S. Agrawal, T. Pakkanen, J.S. Luoma, H. Viita, J. Hartikainen, et al. 1998. Adenovirusmediated gene transfer to lower limb artery of patients with chronic critical leg ischemia. Hum. Gene Ther. 9:1481-1486.

Lamont, R.E., and S. Childs. 2006. MAPping out arteries and veins. Sci. STKE. 2006:pe39.

Lupien, M., J. Eeckhoute, C.A. Meyer, Q. Wang, Y. Zhang, W. Li, J.S. Carroll, X.S. Liu, and M. Brown. 2008. FoxA1 translates epigenetic signatures into enhancer-driven lineage-specific transcription. Cell. 132:958-970.

Macian, F. 2005. NFAT proteins: key regulators of T-cell development and function. Nat. Rev. Immunol. 5:472-484.

Macian, F., C. Lopez-Rodriguez, and A. Rao. 2001. Partners in transcription: NFAT and AP-1. Oncogene. 20:2476-2489.

Makinen, T., T. Veikkola, S. Mustjoki, T. Karpanen, B. Catimel, E.C. Nice, L. Wise, A. Mercer, H. Kowalski, D. Kerjaschki, et al. 2001. Isolated lymphatic endothelial cells transduce growth, survival and migratory signals via the VEGF-C/D receptor VEGFR-3. EMBO J. 20:4762-4773.

Makinen, T., R.H. Adams, J. Bailey, Q. Lu, A. Ziemiecki, K. Alitalo, R. Klein, and G.A. Wilkinson. 2005. PDZ interaction site in ephrinB2 is required for the remodeling of lymphatic vasculature. Genes Dev. 19:397-410

Makinen, T., C. Norrmén, and T.V. Petrova. 2007. Molecular mechanisms of lymphatic vascular development. Cell. Mol. Life Sci. 64:1915-1929.

Mani, S.A., J. Yang, M. Brooks, G. Schwaninger, A. Zhou, N. Miura, J.L. Kutok, K. Hartwell, A.L. Richardson, and R.A. Weinberg. 2007. Mesenchyme Forkhead 1 (FOXC2) plays a key role in metastasis and is associated with aggressive basal-like breast cancers. Proc. Natl. Acad. Sci. USA. 104:10069-10074.

Maretto, S., M. Cordenonsi, S. Dupont, P. Braghetta, V. Broccoli, A.B. Hassan, D. Volpin, G.M. Bressan, and S. Piccolo. 2003. Mapping Wnt/betacatenin signaling during mouse development and in colorectal tumors. Proc. Natl. Acad. Sci. USA. 100:3299-3304.

Matys, V., E. Fricke, R. Geffers, E. Gossling, M. Haubrock, R. Hehl, K. Hornischer, D. Karas, A.E. Kel, O.V. Kel-Margoulis, et al. 2003. TRANSFAC: transcriptional regulation, from patterns to profiles. Nucleic Acids Res. 31:374-378.

Mellor, R.H., G. Brice, A.W. Stanton, J. French, A. Smith, S. Jeffery, J.R Levick, K.G. Burnand, and P.S. Mortimer. 2007. Mutations in FOXC2 are strongly associated with primary valve failure in veins of the lower limb. Circulation. 115:1912-1920.

Meyer, M., M. Clauss, A. Lepple-Wienhues, J. Waltenberger, H.G. Augustin, M. Ziche, C. Lanz, M. Buttner, H.J. Rziha, and C. Dehio. 1999. A novel vascular endothelial growth factor encoded by Orf virus, VEGF-E, mediates angiogenesis via signalling through VEGFR-2 (KDR) but not VEGFR-1 (Flt-1) receptor tyrosine kinases. EMBO J. 18:363-374.

Monticelli, S., and A. Rao. 2002. NFAT1 and NFAT2 are positive regulators of IL-4 gene transcription. Eur. J. Immunol. 32:2971-2978.

Musaro, A., K.J. McCullagh, F.J. Naya, E.N. Olson, and N. Rosenthal. 1999 IGF-1 induces skeletal myocyte hypertrophy through calcineurin in association with GATA-2 and NF-ATc1. Nature. 400:581-585.

O'Geen, H., C.M. Nicolet, K. Blahnik, R. Green, and P.J. Farnham. 2006 Comparison of sample preparation methods for ChIP-chip assays. Biotechniques. 41:577-580.

Ogawa, S., A. Oku, A. Sawano, S. Yamaguchi, Y. Yazaki, and M. Shibuya. 1998 A novel type of vascular endothelial growth factor: VEGF-E (NZ-7 VEGF) preferentially utilizes KDR/Flk-1 receptor and carries a potent mitotic activity without heparin-binding domain. J. Biol. Chem. 273:31273-31282.

Oliver, G. 2004. Lymphatic vasculature development. Nat. Rev. Immunol. 4:35-45.

Petrova, T.V., T. Makinen, T.P. Makela, J. Saarela, I. Virtanen, R.E. Ferrell, D.N. Finegold, D. Kerjaschki, S. Yla-Herttuala, and K. Alitalo. 2002. Lymphatic endothelial reprogramming of vascular endothelial cells by the Prox-1 homeobox transcription factor. EMBO J. 21:4593-4599.

Petrova, T.V., T. Karpanen, C. Norrmén, R. Mellor, T. Tamakoshi, D. Finegold, R. Ferrell, D. Kerjaschki, P. Mortimer, S. Yla-Herttuala, et al. 2004. Defective valves and abnormal mural cell recruitment underlie lymphatic vascular failure in lymphedema distichiasis. Nat. Med. 10:974-981.

Pierrou, S., M. Hellqvist, L. Samuelsson, S. Enerback, and P. Carlsson. 1994 Cloning and characterization of seven human forkhead proteins: binding site specificity and DNA bending. EMBO J. 13:5002-5012.

Ranger, A.M., M.J. Grusby, M.R. Hodge, E.M. Gravallese, F.C. de la Brousse, T. Hoey, C. Mickanin, H.S. Baldwin, and L.H. Glimcher. 1998. The transcription factor NF-ATc is essential for cardiac valve formation. Nature. 392:186-190.

Risau, W. 1997. Mechanisms of angiogenesis. Nature. 386:671-674.

Roulet, E., S. Busso, A.A. Camargo, A.J. Simpson, N. Mermod, and P. Bucher. 2002. High-throughput SELEX SAGE method for quantita- tive modeling of transcription-factor binding sites. Nat. Biotechnol. 20:831-835

Saaristo, A., T. Veikkola, B. Enholm, M. Hytonen, J. Arola, K. Pajusola, P. Turunen, M. Jeltsch, M.J. Karkkainen, D. Kerjaschki, et al. 2002a. Adenoviral VEGF-C overexpression induces blood vessel enlargement, tortuosity, and leakiness but no sprouting angiogenesis in the skin or mucous membranes. FASEB J. 16:1041-1049.

Saaristo, A., T. Veikkola, T. Tammela, B. Enholm, M.J. Karkkainen, K. Pajusola, H. Bueler, S. Yla-Herttuala, and K. Alitalo. 2002b. Lymphangiogenic gene therapy with minimal blood vascular side effects. J. Exp. Med. 196:719-730.

Sakuma, K., J. Nishikawa, R. Nakao, K. Watanabe, T. Totsuka, H. Nakano, M. Sano, and M. Yasuhara. 2003. Calcineurin is a potent regulator for skeletal muscle regeneration by association with NFATc1 and GATA-2. Acta Neuropathol. 105:271-280.

Saleem, R.A., S. Banerjee-Basu, T.C. Murphy, A. Baxevanis, and M.A. Walter. 2004. Essential structural and functional determinants within the forkhead domain of FOXC1. Nucleic Acids Res. 32:4182-4193.

Sato, T.N. 2003. Vascular development: molecular logic for defining arteries and veins. Curr. Opin. Hematol. 10:131-135.

Schulz, R.A., and K.E. Yutzey. 2004. Calcineurin signaling and NFAT activation in cardiovascular and skeletal muscle development. Dev. Biol. 266:1-16.

Shimoda, H., M.J. Bernas, M.H. Witte, N.W. Gale, G.D. Yancopoulos, and S. Kato. 2007. Abnormal recruitment of periendothelial cells to lymphatic capillaries in digestive organs of angiopoietin-2-deficient mice. Cell Tissue Res. 328:329-337.

Siepel, A., G. Bejerano, J.S. Pedersen, A.S. Hinrichs, M. Hou, K. Rosenbloom, H. Clawson, J. Spieth, L.W. Hillier, S. Richards, et al. 2005. Evolutionarily conserved elements in vertebrate, insect, worm, and yeast genomes. Genome Res. 15:1034-1050.

Takayanagi, H. 2007. The role of NFAT in osteoclast formation. Ann. N. Y. Acad. Sci. 1116:227-237.

Tammela, T., A. Saaristo, T. Holopainen, J. Lyytikka, A. Kotronen, M. Pitkonen, U. Abo-Ramadan, S. Yla-Herttuala, T.V. Petrova, and K. Alitalo. 2007. Therapeutic differentiation and maturation of lymphatic vessels after lymph node dissection and transplantation. Nat. Med. 13:1458-1466.

Tammela, T., A. Saaristo, M. Lohela, T. Morisada, J. Tornberg, C. Norrmén, Y. Oike, K. Pajusola, G. Thurston, T. Suda, et al. 2005. Angiopoietin-1 promotes lymphatic sprouting and hyperplasia. Blood. 105:4642-4648.

Thompson, W., E.C. Rouchka, and C.E. Lawrence. 2003. Gibbs recursive sampler: finding transcription factor binding sites. Nucleic Acids Res. 31:3580-3585.

Timmerman, L.A., J.I. Healy, S.N. Ho, L. Chen, C.C. Goodnow, and G.R. Crabtree. 1997. Redundant expression but selective utilization of nuclear factor of activated T cells family members. J. Immunol. 159:2735-2740.

van der Putte, S.C. 1975. The early development of the lymphatic system in mouse embryos. Acta Morphol. Neerl. Scand. 13:245-286.

Veikkola, T., L. Jussila, T. Makinen, T. Karpanen, M. Jeltsch, T.V. Petrova, H. Kubo, G. Thurston, D.M. McDonald, M.G. Achen, et al. 2001. Signalling via vascular endothelial growth factor receptor-3 is sufficient for lymphangiogenesis in transgenic mice. EMBO J. 20:1223-1231.

Veikkola, T., M. Lohela, K. Ikenberg, T. Makinen, T. Korff, A. Saaristo, T. Petrova, M. Jeltsch, H.G. Augustin, and K. Alitalo. 2003. Intrinsic versus microenvironmental regulation of lymphatic endothelial cell phenotype and function. FASEB J. 17:2006-2013.

Wada, H., K. Hasegawa, T. Morimoto, T. Kakita, T. Yanazume, M. Abe, and S. Sasayama. 2002. Calcineurin-GATA-6 pathway is involved in smooth muscle-specific transcription. J. Cell Biol. 156:983-991.

Wick, N., P. Saharinen, J. Saharinen, E. Gurnhofer, C.W. Steiner, I. Raab, D. Stokic, P. Giovanoli, S. Buchsbaum, A. Burchard, et al. 2007. Transcriptomal comparison of human dermal lymphatic endothelial cells ex vivo and in vitro. Physiol. Genomics. 28:179-192.

Wigle, J.T., and G. Oliver. 1999. Prox1 function is required for the development of the murine lymphatic system. Cell. 98:769-778.

Wigle, J.T., N. Harvey, M. Detmar, I. Lagutina, G. Grosveld, M.D. Gunn, D.G. Jackson, and G. Oliver. 2002. An essential role for Prox 1 in the induction of the lymphatic endothelial cell phenotype. EMBO J. 21:1505-1513.

Wilkinson, D.G. 1998. In Situ Hybridization: A Practical Approach. Oxford University Press, Oxford/New York. 224 pp.

Wirzenius, M., T. Tammela, M. Uutela, Y. He, T. Odorisio, G. Zambruno, J.A. Nagy, H.F. Dvorak, S. Yla-Herttuala, M. Shibuya, and K. Alitalo. 2007. Distinct vascular endothelial growth factor signals for lymphatic vessel enlargement and sprouting. J. Exp. Med. 204:1431-1440.

Yang, X.L., H. Matsuura, Y. Fu, T. Sugiyama, and N. Miura. 2000. MFH-1 is required for bone morphogenetic protein-2-induced osteoblastic differentiation of C2C12 myoblasts. FEBS Lett. 470:29-34. 